\title{
An Electromagnetic Crystal Green Function Multiple Scattering Technique for Arbitrary Polarizations, Lattices, and Defects
}

\author{
Davy Pissoort, Eric Michielssen, Fellow, IEEE, and Anthony Grbic, Member, IEEE
}

\begin{abstract}
A generalized electromagnetic crystal (EC) Green function (GF) multiple scattering technique (MST) that permits the simulation of transverse electric and magnetic waves in 2-D EC devices created by replacing crystal cylinders by nonconforming ones is presented. The EC may be defined on a square or triangular lattice. Both EC and nonconforming cylinders can be of arbitrary shape and composition. Integral equations in terms of equivalent currents residing on circular surfaces centered about the nonconforming cylinders are constructed using GFs innate to the background EC. Contrary to the (conventional) free-space GF MST, the proposed generalized EC GF MST yields sparse systems of equations that can be solved efficiently by multifrontal methods. A combination of the generalized EC GF MST with a volume integral-equation- and/or finite-elementbased scheme to calculate scattering matrices of noncircular/ inhomogeneous/plasmonic cylinders yields a very powerful tool that permits simulating wave propagation in a very broad class of EC devices. The generalized EC GF MST is applied to the analysis of a wide variety of practical EC devices, including a third-order Chebyshev bandpass filter, a pair of power dividers, two channel drop filters, a large multiplexer-demultiplexer, a set of bended waveguides, and waveguide filters comprising noncircular or plasmonic cylinders.
\end{abstract}

Index Terms-Numerical analysis, periodic structures, photonic band gap waveguides, photonic crystals.

\section{INTRODUCTION}

$\mathbf{I}$ N RECENT years, many computational schemes for simulating wave propagation in complex 2-D electromagnetic crystal (EC) devices have been proposed (see [1] and references therein). The EC Green function multiple scattering technique (EC GF MST), which is a new integral-equationbased technique presented in [2], distinguishes itself from its predecessors in terms of its computational efficiency and accuracy. The EC GF MST permits the fast analysis of 2-D EC devices that are obtained by "removing" circular crystal elements from doubly periodic, defectless, and infinite ECs comprising identical homogeneous dielectric/magnetic circular cylinders. Just like the conventional free-space GF MST

Manuscript received June 29, 2006; revised October 6, 2006.

D. Pissoort was with the Electromagnetics Group, Department of Information Technology, Ghent University, 9000 Ghent, Belgium. He is now with the Eesof-EDA Department, Agilent Technologies, Ghent, Belgium (e-mail: davy.pissoort@intec.ugent.be).

E. Michielssen and A. Grbic are with the Radiation Laboratory, Department of Electrical Engineering and Computer Science, University of Michigan, Ann Arbor, MI 48109 USA (e-mail: emichiel@umich.edu; agrbic@umich.edu).

Digital Object Identifier 10.1109/JLT.2006.889364
[3], the EC GF MST solves integral equations in terms of equivalent currents that reside on circular cylindrical surfaces. However, contrary to the conventional free-space GF MST, which associates unknowns with the surfaces of all physical cylindrical elements that define the EC device and then subsequently describes their interactions using a free-space GF, the EC GF MST considers equivalent currents that reside on the cylindrical surfaces of the voids left by removed cylinders and then models their interaction via a GF innate to the surrounding infinite EC. For frequencies in the EC band gap, this EC GF can be precomputed easily using the free-space GF MST by considering a centrally excited, finite, and small EC.

When compared to conventional free-space MSTs for analyzing EC devices, the EC GF MST has four advantages of note. All four relate to the nature of the linear system of equations that results upon discretizing the integral equations considered. First, for the vast majority of EC devices, the number of voids/removed cylinders is much smaller than the number of physical cylinders, which translates to smaller linear systems. Second, the EC GF MST's system of equations is essentially sparse, whereas that produced by the free-space GF MST is dense; indeed, for frequencies in the EC band gap, the EC GF decays exponentially with distance, and each and every removed cylinder only interacts with its near neighbors. Third, the EC GF MST's sparse systems of equations can be solved rapidly by multifrontal methods [4], thereby avoiding the excessive iteration counts associated with the iterative solution of the free-space GF MST's dense systems of equations [5]. Fourth, the EC GF MST is ideally suited to extract an EC device's $S$ parameters as it enables the implementation of exact modal excitations and absorbing boundary conditions.

Unfortunately, the formulation of the EC GF MST in [2] was quite narrow, thereby unnecessarily limiting its applicability to EC devices that exactly fit the preceding mold. In reality, many EC devices comprise cylindrical elements that are noncircular, inhomogeneous, plasmonic, etc. In addition, many EC devices cannot be constructed by simply removing cylindrical elements from an otherwise defectless and infinite EC as they contain other types of defects, i.e., elements that do not conform to those of the EC background. In [2], the EC GF MST was applied successfully to EC devices comprising a "small" number of irregular circular defects, e.g., circular cylinders with center positions, radii, and/or material parameters that do not conform to those of the EC background. The EC GFs that reflect the presence of the irregular defects were obtained as 


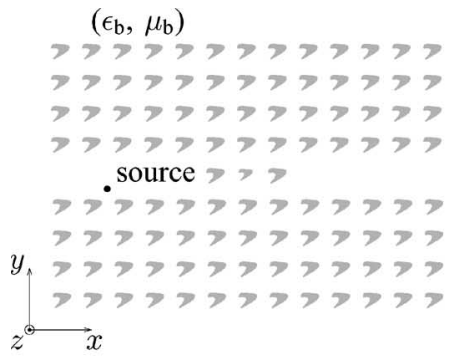

(a)

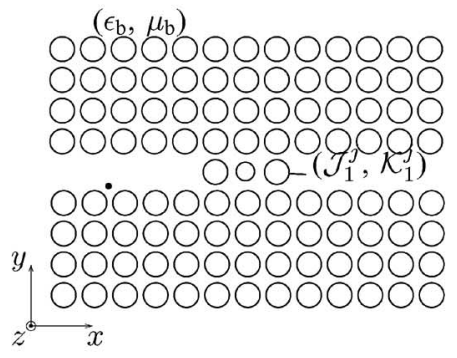

(b)

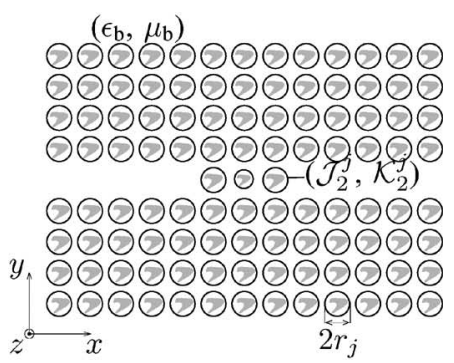

(c)

Fig. 1. Field-equivalence theorem applied to the simulation of an EC device using the EC GF MST. (a) Original configuration. (b) Exterior problem. (c) Interior problem.

low-rank perturbations of the regular EC GF for a removed cylinder. However, for EC devices comprising many irregular defects, the computational complexity of the scheme of [2] is too high. Moreover, the EC GF MST presented in [2] does not apply to EC devices that only comprise irregular defects, e.g., waveguides that are formed by reducing/enlarging the diameter of a row of cylinders [6].

This paper describes a "generalized" EC GF MST that broadens the scheme's applicability to virtually arbitrary EC configurations and excitations while retaining the original scheme's benefits. Specifically, the proposed scheme efficiently handles EC devices that are defined on square or triangular lattices supporting both transverse magnetic and electric polarized fields and that are obtained by "replacing" cylindrical elements from a defectless EC with cylindrical elements that "do not conform to those of the EC background." These cylindrical elements can be of arbitrary shape and composition. Contrary to the scheme of [2], which considers only equivalent currents on circular cylindrical surfaces centered about "removed cylindrical elements," the generalized scheme considers equivalent currents on circular cylindrical surfaces centered around "all defects." Although for every type of defect, another type of EC GF is required, the precomputation of all these GFs can be achieved rapidly once a few template EC GFs are known. If combined with volume integral-equation- and/or finite-element-based schemes (e.g., the unimoment method [7], [8]) to calculate the scattering matrix of arbitrarily shaped and/or composed cylindrical elements, the proposed generalized scheme yields a very powerful tool that permits the fast simulation of wave propagation in a very broad class of 2-D EC devices.

This paper is organized as follows: Section II describes a generalized free-space GF MST for characterizing EC devices comprising arbitrarily positioned and arbitrarily shaped dielectric/magnetic cylinders. Section III describes the generalized EC GF MST for characterizing EC devices obtained by replacing an arbitrary number of cylindrical elements from a defectless EC by nonconforming ones. Section IV shows how all required GFs easily can be obtained without resorting to solving a new linear system of equations for each and every defect considered. Section V elucidates the versatility of the proposed scheme via its application to a third-order Chebyshev bandpass filter, a pair of power dividers, two channel drop filters, a large multiplexer-demultiplexer, a set of bended waveguides, and three EC waveguide filters comprising noncircular or plasmonic cylinders. Finally, Section VI summarizes the pro- posed scheme's principal features and proffers suggestions for future research.

\section{Analysis of EC Devices Using a Generalized FREE-SPACE GF MST}

This section details a generalized free-space GF MST [2], [3] for characterizing finite EC devices that comprise arbitrarily positioned and arbitrarily shaped dielectric/magnetic cylindrical elements. First, it is shown that by casting Maxwell's equations in terms of generalized fields, currents, and constitutive parameters, the proposed algorithm applies to both transverse magnetic and electric to $z\left(\mathrm{TM}_{z}\right.$ and $\left.\mathrm{TE}_{z}\right)$ fields. Starting from the field-equivalence principle [9], two sets of integral equations in terms of equivalent electric and magnetic currents residing on circular cylindrical surfaces centered about all physical cylindrical elements are derived. The second (interior) set of integral equations permits the elimination of one type of current. For circular and homogeneous cylindrical elements, this relationship can be found in closed form. For arbitrarily shaped and/or composed elements, this relationship has to be established via a volume integral-equation- or finite-elementbased method. Upon substituting this relationship in the first (exterior) set of integral equations, the free-space GF is defined, and a dense linear system of equations is obtained.

Consider a finite 2-D EC device comprising $N_{c}$ infinite $z$-invariant dielectric/magnetic cylindrical elements residing in a homogeneous background medium with constitutive parameters $\left(\epsilon_{b}, \mu_{b}\right)$ (permittivity, permeability) [Fig. 1(a)]. The cylindrical elements can be of arbitrary shape and material composition. The following nomenclature and terminology are used.

- All sources and fields are assumed to be time harmonic with angular frequency $\omega$; temporal dependencies $e^{\jmath \omega t}(\jmath=\sqrt{-1})$ are suppressed.

- $\boldsymbol{\rho},(\rho, \phi)$, and $(\hat{\boldsymbol{\rho}}, \hat{\boldsymbol{\phi}})$ denote the global position vectors, cylindrical coordinates, and cylindrical unit vectors, respectively.

- $\boldsymbol{\rho}_{j}^{c}$ and $\left(\rho_{j}^{c}, \phi_{j}^{c}\right)$ denote the position vector and cylindrical coordinates of the center of cylindrical element $j, j=$ $1, \ldots, N_{c}$, respectively.

- $\boldsymbol{\rho}_{j},\left(\rho_{j}, \phi_{j}\right)$, and $\left(\hat{\boldsymbol{\rho}}_{j}, \hat{\boldsymbol{\phi}}_{j}\right)$ denote the position vector, cylindrical coordinates, and cylindrical unit vectors in the coordinate system centered about $\boldsymbol{\rho}_{j}^{c}$, respectively. 
- $(\epsilon(\boldsymbol{\rho})$ and $\mu(\boldsymbol{\rho}))$ denote the permittivity and permeability at $\rho$, respectively.

- $k_{b}=\omega \sqrt{\epsilon_{b} \mu_{b}}$ represents the wavenumber in the homogeneous background medium.

- $r_{j}, j=1, \ldots, N_{c}$ is the radius of the smallest circle centered about $\boldsymbol{\rho}_{j}^{c}$ circumscribing circular element $j$.

Let $\boldsymbol{E}^{\text {inc }}(\boldsymbol{\rho})$ and $\boldsymbol{H}^{\text {inc }}(\boldsymbol{\rho})$ denote the incident electric and magnetic fields radiated by impressed sources (assumed to reside outside all cylindrical elements) in the absence of any cylindrical elements, respectively. In the presence of the cylindrical elements, the total fields $\boldsymbol{E}^{\text {tot }}(\boldsymbol{\rho})$ and $\boldsymbol{H}^{\text {tot }}(\boldsymbol{\rho})$ are observed. The difference between the total and incident fields defines the scattered fields $\boldsymbol{E}^{\text {sca }}(\boldsymbol{\rho})$ and $\boldsymbol{H}^{\text {sca }}(\boldsymbol{\rho})$. To describe the total and scattered fields, two sets $(\alpha=1,2)$ of equivalent electric and magnetic currents $\left(\mathcal{J}_{\alpha}^{j}(\rho), \mathcal{K}_{\alpha}^{j}(\rho)\right)$ residing on the circular surface $S_{j}$ with radius $r_{j}$ centered about $\boldsymbol{\rho}_{j}^{c}$, $j=1, \ldots, N_{c}$ are introduced [Fig. 1(b) and (c)]. These currents relate to the total fields on $S_{j}$ as

$$
\begin{aligned}
& \mathcal{J}_{\boldsymbol{\alpha}}^{\boldsymbol{j}}(\boldsymbol{\rho})=s_{\alpha} \hat{\boldsymbol{\rho}}_{j} \times\left.\boldsymbol{H}^{\mathrm{tot}}(\boldsymbol{\rho})\right|_{\boldsymbol{\rho} \in S_{j}} \delta\left(\rho_{j}-r_{j}\right) \\
& \mathcal{K}_{\boldsymbol{\alpha}}^{\boldsymbol{j}}(\boldsymbol{\rho})=-s_{\alpha} \hat{\boldsymbol{\rho}}_{j} \times\left.\boldsymbol{E}^{\mathrm{tot}}(\boldsymbol{\rho})\right|_{\boldsymbol{\rho} \in S_{j}} \delta\left(\rho_{j}-r_{j}\right)
\end{aligned}
$$

with $s_{1}=1$ and $s_{2}=-1$. To analyze wave propagation in 2-D EC devices, it suffices to consider the two noninteracting $\mathrm{TM}_{z}$ and $\mathrm{TE}_{z}$ polarizations. Because of duality, the same formulation applies to both polarizations if Maxwell's equations are cast in terms of generalized fields $\left(\boldsymbol{F}^{\boldsymbol{\tau}}(\boldsymbol{\rho}), \boldsymbol{G}^{\boldsymbol{\tau}}(\boldsymbol{\rho})\right)(\tau=$ inc, tot, sca), currents $\left(\mathcal{P}_{\alpha}^{j}(\rho), \mathcal{Q}_{\alpha}^{j}(\rho)\right)$, and constitutive parameters $(\kappa(\boldsymbol{\rho}), \theta(\boldsymbol{\rho}))$. For the $\mathrm{TM}_{z}$ polarization, these generalized fields, currents, and constitutive parameters are

$$
\begin{aligned}
\boldsymbol{F}^{\boldsymbol{\tau}}(\boldsymbol{\rho}) & =F^{\tau}(\boldsymbol{\rho}) \hat{\boldsymbol{z}}=E^{\tau}(\boldsymbol{\rho}) \hat{\boldsymbol{z}} \\
\boldsymbol{G}^{\boldsymbol{\tau}}(\boldsymbol{\rho}) & =G_{\rho_{j}}^{\tau}(\boldsymbol{\rho}) \hat{\boldsymbol{\rho}}_{j}+G_{\phi_{j}}^{\tau}(\boldsymbol{\rho}) \hat{\boldsymbol{\phi}}_{j} \\
& =H_{\rho_{j}}^{\tau}(\boldsymbol{\rho}) \hat{\boldsymbol{\rho}}_{j}+H_{\phi_{j}}^{\tau}(\boldsymbol{\rho}) \hat{\boldsymbol{\phi}}_{j} \\
\mathcal{P}_{\boldsymbol{\alpha}}^{j}(\boldsymbol{\rho}) & =\mathcal{P}_{\alpha}^{j}(\boldsymbol{\rho}) \hat{\boldsymbol{z}}=\mathcal{J}_{\alpha}^{j}(\boldsymbol{\rho}) \hat{\boldsymbol{z}} \\
\mathcal{Q}_{\boldsymbol{\alpha}}^{j}(\boldsymbol{\rho}) & =\mathcal{Q}_{\alpha}^{j}(\boldsymbol{\rho}) \hat{\boldsymbol{\phi}}_{j}=\mathcal{K}_{\alpha}^{j}(\boldsymbol{\rho}) \hat{\boldsymbol{\phi}}_{j} \\
\kappa(\boldsymbol{\rho}) & =\epsilon(\boldsymbol{\rho}) \\
\theta(\boldsymbol{\rho}) & =\mu(\boldsymbol{\rho})
\end{aligned}
$$

while for the $\mathrm{TE}_{z}$ polarization, they are

$$
\begin{aligned}
\boldsymbol{F}^{\boldsymbol{\tau}}(\boldsymbol{\rho}) & =F^{\tau}(\boldsymbol{\rho}) \hat{\boldsymbol{z}}=-H^{\tau}(\boldsymbol{\rho}) \hat{\boldsymbol{z}} \\
\boldsymbol{G}^{\boldsymbol{\tau}}(\boldsymbol{\rho}) & =G_{\rho_{j}}^{\tau}(\boldsymbol{\rho}) \hat{\boldsymbol{\rho}}_{j}+G_{\phi_{j}}^{\tau}(\boldsymbol{\rho}) \hat{\boldsymbol{\phi}}_{j} \\
& =E_{\rho_{j}}^{\tau}(\boldsymbol{\rho}) \hat{\boldsymbol{\rho}}_{j}+E_{\phi_{j}}^{\tau}(\boldsymbol{\rho}) \hat{\boldsymbol{\phi}}_{j} \\
\mathcal{P}_{\boldsymbol{\alpha}}^{j}(\boldsymbol{\rho}) & =\mathcal{P}_{\alpha}^{j}(\boldsymbol{\rho}) \hat{\boldsymbol{z}}=-\mathcal{K}_{\alpha}^{j}(\boldsymbol{\rho}) \hat{\boldsymbol{z}} \\
\mathcal{Q}_{\boldsymbol{\alpha}}^{j}(\boldsymbol{\rho}) & =\mathcal{Q}_{\alpha}^{j}(\boldsymbol{\rho}) \hat{\boldsymbol{\phi}}_{j}=\mathcal{J}_{\alpha}^{j}(\boldsymbol{\rho}) \hat{\boldsymbol{\phi}}_{j} \\
\kappa(\boldsymbol{\rho}) & =\mu(\boldsymbol{\rho}) \\
\theta(\boldsymbol{\rho}) & =\epsilon(\boldsymbol{\rho}) .
\end{aligned}
$$

All other field components vanish. From here on forward, the generalized free-space and EC GF MST will be described in terms of these generalized fields, currents, and constitutive parameters, thereby simultaneously covering both the $\mathrm{TM}_{z}$ and $\mathrm{TE}_{z}$-polarizations.

The following two facts are derived from the fieldequivalence theorem (Fig. 1):

1) The currents $\sum_{j=1}^{N_{c}} \mathcal{P}_{1}^{j}(\boldsymbol{\rho}) \hat{\boldsymbol{z}}$ and $\sum_{j=1}^{N_{c}} \mathcal{Q}_{1}^{j}(\boldsymbol{\rho}) \hat{\boldsymbol{\phi}}_{j}$, when radiating alongside the impressed sources in an unbounded medium with constitutive parameters $\left(\kappa_{b}, \theta_{b}\right)$, generate zero fields inside each surface $S_{j}$ centered about $\boldsymbol{\rho}_{j}^{c}$ and the total fields $F^{\text {tot }}(\boldsymbol{\rho}) \hat{\boldsymbol{z}}$ and $G_{\rho_{j}}^{\text {tot }}(\boldsymbol{\rho}) \hat{\boldsymbol{\rho}}_{j}+$ $\tilde{G}_{\phi_{j}}^{\text {tot }}(\rho) \hat{\phi}_{j}$ outside all $S_{j}$.

2) The currents $\mathcal{P}_{2}^{j}(\boldsymbol{\rho}) \hat{\boldsymbol{z}}$ and $\mathcal{Q}_{2}^{j}(\boldsymbol{\rho}) \hat{\boldsymbol{\phi}}_{j}$, when radiating around cylindrical element $j$ placed in an unbounded medium with constitutive parameters $\left(\kappa_{b}, \theta_{b}\right)$, generate zero fields outside $S_{j}$ and $F^{\text {tot }}(\boldsymbol{\rho}) \hat{\boldsymbol{z}}$ and $G_{\rho_{j}}^{\text {tot }}(\boldsymbol{\rho}) \hat{\boldsymbol{\rho}}_{j}+$ $\tilde{G}_{\phi_{j}}^{\text {tot }}(\boldsymbol{\rho}) \hat{\boldsymbol{\phi}}_{j}$ inside $S_{j}$.

If $F_{\alpha}^{\mathrm{sca}, j}(\boldsymbol{\rho}) \hat{\boldsymbol{z}}$ denotes the $z$-directed field radiated jointly by $\mathcal{P}_{\alpha}^{j}(\boldsymbol{\rho}) \hat{\boldsymbol{z}}$ and $\mathcal{Q}_{\alpha}^{j}(\boldsymbol{\rho}) \hat{\boldsymbol{\phi}}_{j}$ in the homogeneous medium with constitutive parameters $\left(\kappa_{b}, \theta_{b}\right)$ when $\alpha=1$, or radiated by $\mathcal{P}_{\alpha}^{j} \hat{z}$ and $\mathcal{Q}_{\alpha}^{j} \hat{\phi}_{j}$ around the irregular cylinder $j$ placed in the unbounded medium with constitutive parameters $\left(\kappa_{b}, \theta_{b}\right)$ when $\alpha=2$, then the preceding statements can be cast as

$$
\begin{aligned}
& F^{\mathrm{inc}}(\boldsymbol{\rho})+\sum_{j=1}^{N_{c}} F_{1}^{\mathrm{sca}, j}(\boldsymbol{\rho})=0 \quad \text { if } \quad \boldsymbol{\rho} \in S_{i}^{-}, i=1, \ldots, N_{c} \\
& F_{2}^{\mathrm{sca}, j}(\boldsymbol{\rho})=0 \quad \text { if } \quad \boldsymbol{\rho} \in S_{j}^{+}, j=1, \ldots, N_{c} .
\end{aligned}
$$

Here, $S_{j}^{-}$and $S_{j}^{+}$denote surfaces residing just inside and outside surface $S_{j}$, respectively. To solve (15) and (16), $\mathcal{P}_{\alpha}^{j}(\boldsymbol{\rho})$ and $\mathcal{Q}_{\alpha}^{j}(\boldsymbol{\rho})$ are expanded as

$$
\begin{aligned}
& \mathcal{P}_{\alpha}^{j}(\boldsymbol{\rho})=s_{\alpha} \sum_{n=-K}^{K} \frac{P_{n}^{j}}{2 \pi r_{j}} e^{j n \phi_{j}} \delta\left(\rho_{j}-r_{j}\right) \\
& \mathcal{Q}_{\alpha}^{j}(\boldsymbol{\rho})=s_{\alpha} \sum_{n=-K}^{K} \frac{Q_{n}^{j}}{2 \pi r_{j}} e^{j n \phi_{j}} \delta\left(\rho_{j}-r_{j}\right) .
\end{aligned}
$$

Because $r_{j}$ is small compared with the wavelength and because the cylindrical elements are assumed sufficiently separated from one another as well as from the impressed sources, the range of the modal index can always be restricted to $n=$ $-K, \ldots, K$, with $K$ being a small positive integer. As detailed in the Appendix, solving (interior problem) (16) yields

$$
Q_{m}^{j}=\sum_{n=-K}^{K} \mathcal{Y}_{m n}^{j} P_{n}^{j} \quad \text { or } \quad \boldsymbol{Q}^{j}=\mathcal{Y}^{j} \boldsymbol{P}^{j} .
$$

This relationship permits $F_{1}^{\text {sca, } j}(\boldsymbol{\rho}) \hat{\boldsymbol{z}}$, which is the $z$-directed field radiated jointly by $\mathcal{P}_{1}^{j}(\boldsymbol{\rho}) \hat{\boldsymbol{z}}$ and $\mathcal{Q}_{1}^{j}(\boldsymbol{\rho}) \hat{\phi}_{j}$ in the homogeneous medium with constitutive parameters $\left(\kappa_{b}, \theta_{b}\right)$, to be expressed as

$$
F_{1}^{\mathrm{sca}, j}(\boldsymbol{\rho})=\sum_{n=-K}^{K} \mathcal{G}_{n}^{j}\left(\boldsymbol{\rho}_{j}\right) P_{n}^{j} .
$$

Quantity $\mathcal{G}_{n}^{j}\left(\boldsymbol{\rho}_{j}\right)$ is the (generalized) free-space GF for a Huygens source with distributed components 


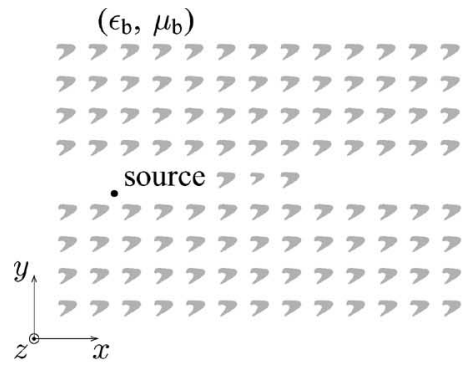

(a)

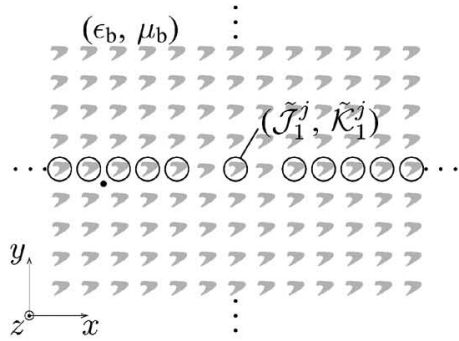

(b) $\left(\epsilon_{\mathrm{b}}, \mu_{\mathrm{b}}\right)$

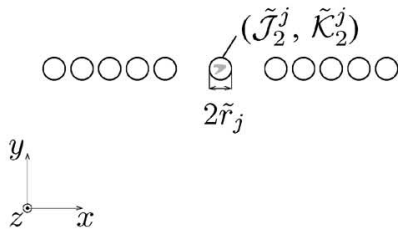

(c)

Fig. 2. Field-equivalence theorem applied to the simulation of an EC device using the EC GF MST. (a) Original configuration. (b) Exterior problem. (c) Interior problem.

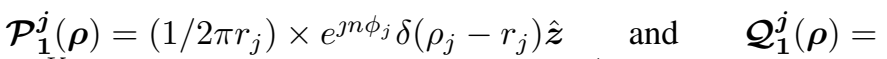
$\sum_{m=-K}^{K}\left(\mathcal{Y}_{m n}^{j} / 2 \pi r_{j}\right) \times e^{j m \phi_{j}} \delta\left(\rho_{j}-r_{j}\right) \hat{\boldsymbol{\phi}}_{j}$, radiating jointly in the homogeneous medium with constitutive parameters $\left(\kappa_{b}, \theta_{b}\right)$. It is readily verified that $\mathcal{G}_{n}^{j}\left(\boldsymbol{\rho}_{j}\right)$ can be expressed as in (21), shown at the bottom of the page, where $J_{n}(\cdot)$ is the $n$ th-order Bessel function of the first kind, and $H_{n}^{(2)}(\cdot)$ is the $n$ th-order Hankel function of the second kind.

To solve for the unknown $P_{n}^{j}$, expansion (20) is inserted into (15), and the resulting equation is tested by

$$
T_{m}^{i}(\boldsymbol{\rho})=\frac{1}{2 \pi r_{i} J_{m}\left(k_{b} r_{i}\right)} e^{-\jmath m \phi_{i}} \delta\left(\rho_{i}-r_{i}\right)
$$

for $i=1, \ldots, N_{c}$ and $n=-K, \ldots, K$, resulting in the matrix equation

$$
Z P=F
$$

where $Z_{m n}^{i j}=\left\langle T_{m}^{i}(\boldsymbol{\rho}), \mathcal{G}_{n}^{j}\left(\boldsymbol{\rho}_{j}\right)\right\rangle$ and $F_{m}^{i}=\left\langle T_{m}^{i}(\boldsymbol{\rho}),-F^{\text {inc }}(\boldsymbol{\rho})\right\rangle$.

\section{Analysis of EC Devices Using the GENERALIZED EC GF MST}

This section details the proposed generalized EC GF MST for the characterization of semi-infinite EC devices that are obtained by "replacing" cylindrical elements from an otherwise infinitely periodic and defectless EC by nonconforming ones. Starting from the field-equivalence principle, two sets of integral equations in terms of equivalent electric and magnetic currents residing on circular cylindrical surfaces centered about only the defects are derived. The second (interior) set of integral equations permits the elimination of one type of current. Upon substituting this relationship in the first (exterior) set of integral equations, the EC GF is defined, and a sparse linear system of equations is obtained.

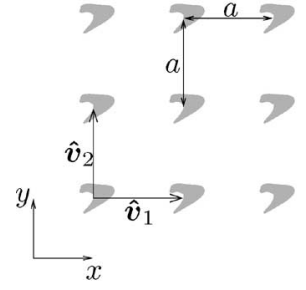

(a)

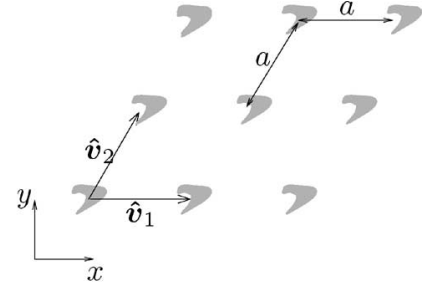

(b)
Fig. 3. Definition of the lattice vectors. (a) Square lattice. (b) Triangular lattice.

Consider a 2-D EC device obtained by replacing $N_{r}$ cylindrical elements from an infinite and defectless EC by cylindrical elements that do not conform to those of the EC background [Fig. 2(a)]. These elements henceforth will be termed "irregular." Two types of irregular elements are defined: "Type-I" irregular elements comprise only removed elements, and "Type-II" irregular elements comprise all other types of defects. The background EC comprises identical, infinite, and $z$-invariant dielectric/magnetic cylindrical elements that snap to a regular square or triangular lattice (with lattice constant $a$ ) and that reside in a homogeneous background medium with constitutive parameters $\left(\epsilon_{b}, \mu_{b}\right)$. The nomenclature in this section adheres to that of Section II, with the exception that tildes appear on symbols referring to fields, currents, and surfaces centered about irregular cylindrical elements.

Furthermore, the following additional assumptions are made, and the following nomenclature/terminology is used.

- The angular frequency $\omega$ is assumed to lie within the EC band gap.

- $\left(\hat{\boldsymbol{v}}_{1}, \hat{\boldsymbol{v}}_{2}\right)$ denotes lattice vectors (Fig. 3) that relate to the Cartesian unit vectors $(\hat{\boldsymbol{x}}, \hat{\boldsymbol{y}})$ as

$$
\begin{aligned}
& \hat{\boldsymbol{v}}_{1}=a \hat{\boldsymbol{x}} \\
& \hat{\boldsymbol{v}}_{2}=a \hat{\boldsymbol{y}}
\end{aligned}
$$

$$
\mathcal{G}_{n}^{j}\left(\boldsymbol{\rho}_{j}\right)=- \begin{cases}{\left[\frac{\omega \theta_{b}}{4} J_{n}\left(k_{b} r_{j}\right)+\frac{\jmath k_{b}}{4} \sum_{p=-K}^{K} \mathcal{Y}_{p n} J_{p}^{\prime}\left(k_{b} r_{j}\right)\right] H_{n}^{(2)}\left(k_{b} \rho_{j}\right) e^{j n \phi_{j}},} & \text { if } \rho_{j}>r \\ {\left[\frac{\omega \theta_{b}}{4} H_{n}^{(2)}\left(k_{b} r_{j}\right)+\frac{\jmath k_{b}}{4} \sum_{p=-K}^{K} \mathcal{Y}_{p n} H_{p}^{(2)^{\prime}}\left(k_{b} r_{j}\right)\right] J_{n}\left(k_{b} \rho_{j}\right) e^{j n \phi_{j}},} & \text { if } \rho_{j}<r\end{cases}
$$


for a square lattice and as

$$
\begin{aligned}
& \hat{\boldsymbol{v}}_{1}=a \hat{\boldsymbol{x}} \\
& \hat{\boldsymbol{v}}_{2}=\frac{a}{2} \hat{\boldsymbol{x}}+\frac{\sqrt{3} a}{2} \hat{\boldsymbol{y}}
\end{aligned}
$$

for a triangular lattice.

- $\rho_{l_{1} l_{2}}^{c}$ and $\left(\rho_{l_{1} l_{2}}^{c}, \phi_{l_{1} l_{2}}^{c}\right)$ denote the position vectors and cylindrical coordinates of the lattice sites, viz. $\rho_{l_{1} l_{2}}^{c}=$ $l_{1} \boldsymbol{v}_{1}+l_{2} \boldsymbol{v}_{2}, l_{1}, l_{2}=\ldots,-1,0,1, \ldots$

- $r$ is the radius of the smallest circle centered about a lattice site and circumscribing the corresponding regular cylindrical element.

- $\tilde{r}_{j}, j=1, \ldots, N_{r}$ is the maximum of $r$ and the radius of the smallest circle centered about a lattice site and circumscribing the irregular cylindrical element $j$.

Let $\tilde{\boldsymbol{F}}^{\text {inc }}(\boldsymbol{\rho})$ and $\tilde{\boldsymbol{G}}^{\text {inc }}(\boldsymbol{\rho})$ denote (generalized) incident fields generated by impressed sources (assumed to reside outside all cylindrical elements) that radiate "in the presence of the defectless EC," viz. the infinite EC "without any irregular cylindrical elements." Upon replacing the cylindrical elements, total fields $\tilde{\boldsymbol{F}}^{\text {tot }}(\boldsymbol{\rho})$ and $\tilde{\boldsymbol{G}}^{\text {tot }}(\boldsymbol{\rho})$ are observed. The difference between the total and incident fields defines the scattered fields $\tilde{\boldsymbol{F}}^{\text {sca }}(\boldsymbol{\rho})$ and $\tilde{\boldsymbol{G}}^{\text {sca }}(\boldsymbol{\rho})$. To describe the total and scattered fields, two sets $(\alpha=1,2)$ of equivalent (generalized) currents $\left(\tilde{\mathcal{P}}_{\boldsymbol{\alpha}}^{j}(\boldsymbol{\rho})\right.$, $\left.\tilde{\mathcal{Q}}_{\alpha}^{j}(\rho)\right)$ residing on the circular surface $\tilde{S}_{j}$ with radius $\tilde{r}_{j}$ centered about $\rho_{l_{1}^{j} l_{2}^{j}}^{c}, j=1, \ldots, N_{r}$ are introduced [Fig. 2(b) and (c)]. These currents relate to the total fields on $\tilde{S}_{j}$ as

$$
\begin{aligned}
& \tilde{\mathcal{P}}_{\boldsymbol{\alpha}}^{j}(\boldsymbol{\rho})=s_{\alpha} \hat{\boldsymbol{\rho}}_{l_{1}^{j} l_{2}^{j}} \times\left.\tilde{\boldsymbol{G}}^{\mathrm{tot}}(\boldsymbol{\rho})\right|_{\boldsymbol{\rho} \in \tilde{S}_{j}} \delta\left(\rho_{l_{1}^{j} l_{2}^{j}}-\tilde{r}_{j}\right) \\
& \tilde{\mathcal{Q}}_{\boldsymbol{\alpha}}^{j}(\boldsymbol{\rho})=-s_{\alpha} \hat{\boldsymbol{\rho}}_{l_{1}^{j} l_{2}^{j}} \times\left.\tilde{F}^{\mathrm{tot}}(\boldsymbol{\rho})\right|_{\boldsymbol{\rho} \in \tilde{S}_{j}} \delta\left(\rho_{l_{1}^{j} l_{2}^{j}}-\tilde{r}_{j}\right)
\end{aligned}
$$

with $s_{1}=1$ and $s_{2}=-1$.

The following two facts are derived from the fieldequivalence theorem (Fig. 2):

1) The currents $\sum_{j=1}^{N_{r}} \tilde{\mathcal{P}}_{1}^{j}(\boldsymbol{\rho}) \hat{\boldsymbol{z}}$ and $\sum_{j=1}^{N_{r}} \tilde{\mathcal{Q}}_{1}^{j}(\boldsymbol{\rho}) \hat{\boldsymbol{\phi}}_{l_{1}^{j} l_{2}^{j}}$, when radiating alongside the impressed sources "in the unbounded" and "defectless EC," generate zero fields inside the surface $\tilde{S}_{j}$ centered about $\boldsymbol{\rho}_{l_{1}^{j} l_{2}^{j}}^{c}$ and the total fields $\tilde{F}^{\text {tot }}(\boldsymbol{\rho}) \hat{\boldsymbol{z}}$ and $\tilde{G}_{\rho_{l_{1}^{j} l_{2}^{j}}}^{\text {tot }}(\boldsymbol{\rho}) \hat{\boldsymbol{\rho}}_{l_{1}^{j} l_{2}^{j}}+\tilde{G}_{\phi_{l_{1}^{j} l_{2}^{j}}}^{\text {tot }}(\boldsymbol{\rho}) \hat{\boldsymbol{\phi}}_{l_{1}^{j} l_{2}^{j}}$ outside all $\tilde{S}_{j}$.

2) The currents $\tilde{\mathcal{P}}_{2}^{j}(\boldsymbol{\rho}) \hat{\boldsymbol{z}}$ and $\tilde{\mathcal{Q}}_{2}^{j}(\boldsymbol{\rho}) \hat{\boldsymbol{\phi}}_{l_{1}^{j} l_{2}^{j}}$, when radiating around the irregular cylindrical element $j$ placed in an unbounded medium with constitutive parameters $\left(\kappa_{b}, \theta_{b}\right)$, generate zero fields outside $\tilde{S}_{j}$ and $\tilde{F}^{\text {tot }}(\boldsymbol{\rho}) \hat{\boldsymbol{z}}$ and $\tilde{G}_{\rho_{l_{1}^{j} l_{2}^{j}}}^{\text {tot }}(\boldsymbol{\rho}) \hat{\boldsymbol{\rho}}_{l_{1}^{j} l_{2}^{j}}+\tilde{G}_{\phi_{l_{1}^{j} l_{2}^{j}}}^{\text {tot }}(\boldsymbol{\rho}) \hat{\boldsymbol{\phi}}_{l_{1}^{j} l_{2}^{j}}$ inside $\tilde{S}_{j}$.

If $\tilde{F}_{\alpha}^{\text {sca, } j}(\boldsymbol{\rho}) \hat{\boldsymbol{z}}$ denotes the $z$-directed field radiated jointly by $\tilde{\mathcal{P}}_{\alpha}^{j}(\boldsymbol{\rho}) \hat{\boldsymbol{z}}$ and $\tilde{\mathcal{Q}}_{\alpha}^{j}(\boldsymbol{\rho}) \hat{\boldsymbol{\phi}}_{l_{1}^{j} l_{2}^{j}}$ in the unbounded and defectless EC when $\alpha=1$, or radiated by $\tilde{\mathcal{P}}_{\alpha}^{j} \hat{z}$ and $\tilde{\mathcal{Q}}_{\alpha}^{j} \hat{\boldsymbol{\phi}}_{l_{1}^{j} l_{2}^{j}}$ around the irregular cylindrical element $j$ placed in the unbounded medium with constitutive parameters $\left(\kappa_{b}, \theta_{b}\right)$ when $\alpha=2$, then the preceding statements can be cast as

$$
\tilde{F}^{\mathrm{inc}}(\boldsymbol{\rho})+\sum_{j=1}^{N_{r}} \tilde{F}_{1}^{\mathrm{sca}, j}(\boldsymbol{\rho})=0 \quad \text { if } \quad \boldsymbol{\rho} \in \tilde{S}_{i}^{-}, i=1, \ldots, N_{r}
$$

$$
\tilde{F}_{2}^{\text {sca }, j}(\boldsymbol{\rho})=0 \quad \text { if } \quad \boldsymbol{\rho} \in \tilde{S}_{j}^{+}, j=1, \ldots, N_{r} .
$$

Here, $\tilde{S}_{j}^{-}$and $\tilde{S}_{j}^{+}$denote surfaces residing just inside and outside surface $\tilde{S}_{j}$, respectively. To solve (30) and (31), $\tilde{\mathcal{P}}_{\alpha}^{j}(\boldsymbol{\rho})$ and $\tilde{\mathcal{Q}}_{\alpha}^{j}(\boldsymbol{\rho})$ are expanded as

$$
\begin{aligned}
& \tilde{\mathcal{P}}_{\alpha}^{j}(\boldsymbol{\rho})=s_{\alpha} \sum_{n=-K}^{K} \frac{\tilde{P}_{n}^{j}}{2 \pi \tilde{r}_{j}} e^{j n \phi_{l_{1}^{j} l_{2}^{j}} \delta\left(\rho_{l_{1}^{j} l_{2}^{j}}-\tilde{r}_{j}\right)} \\
& \tilde{\mathcal{Q}}_{\alpha}^{j}(\boldsymbol{\rho})=s_{\alpha} \sum_{n=-K}^{K} \frac{\tilde{Q}_{n}^{j}}{2 \pi \tilde{r}_{j}} e^{j n \phi_{l_{1}^{j} l_{2}^{j}} \delta\left(\rho_{l_{1}^{j} l_{2}^{j}}-\tilde{r}_{j}\right) .}
\end{aligned}
$$

Just like in the generalized free-space GF MST, solving (interior problem) (31) yields

$$
\tilde{Q}_{m}^{j}=\sum_{n=-K}^{K} \mathcal{Y}_{m n}^{j} \tilde{P}_{n}^{j} \quad \text { or } \quad \tilde{\boldsymbol{Q}}^{j}=\mathcal{Y}^{j} \tilde{\boldsymbol{P}}^{j}
$$

This relationship permits $\tilde{F}_{1}^{\text {sca, } j}(\boldsymbol{\rho}) \hat{z}$, which is the $z$-directed field radiated jointly by $\tilde{\mathcal{P}}_{1}^{j}(\boldsymbol{\rho}) \hat{\boldsymbol{z}}$ and $\tilde{\mathcal{Q}}_{1}^{j}(\boldsymbol{\rho}) \hat{\boldsymbol{\phi}}_{l_{1}^{j} l_{2}^{j}}$ "in the defectless" and "unbounded EC," to be expressed as

$$
\tilde{F}_{1}^{\mathrm{sca}, j}(\boldsymbol{\rho})=\sum_{n=-K}^{K} \tilde{\mathcal{G}}_{n}^{j}\left(\boldsymbol{\rho}_{l_{1}^{j} l_{2}^{j}}\right) \tilde{P}_{n}^{j}
$$

Quantity $\tilde{\mathcal{G}}_{n}^{j}\left(\boldsymbol{\rho}_{l_{1}^{j} l_{2}^{j}}\right)$ is the (generalized) EC GF for a Huygens source with distributed components $\tilde{\mathcal{P}}_{1}^{j}(\boldsymbol{\rho})=$

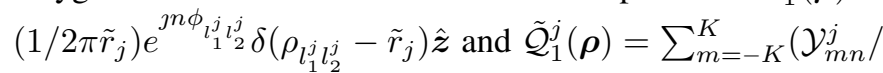
$\left.2 \pi \tilde{r}_{j}\right) e^{j m \phi_{l_{1}^{j}} l_{2}^{j}} \delta\left(\rho_{l_{1}^{j} l_{2}^{j}}-\tilde{r}_{j}\right) \hat{\boldsymbol{\phi}}_{l_{1}^{j} l_{2}^{j}}$, radiating jointly in the "defectless" and "unbounded EC." Contrary to the free-space $\mathrm{GF}$, no closed-form expression for $\tilde{\mathcal{G}}_{n}^{j}\left(\boldsymbol{\rho}_{l_{1}^{j} l_{2}^{j}}\right)$ exists.

To solve for the unknown $\tilde{P}_{n}^{j}$, expansion (35) is inserted into (30), and the resulting equation is tested by

$$
\tilde{T}_{m}^{i}(\boldsymbol{\rho})=\frac{1}{2 \pi \tilde{r}_{i} J_{m}\left(k_{b} \tilde{r}_{i}\right)} e^{-\jmath m \phi_{l_{1}^{i} l_{2}^{i}} \delta}\left(\rho_{l_{1}^{i} l_{2}^{i}}-\tilde{r}_{i}\right)
$$

for $i=1, \ldots, N_{r}$ and $n=-K, \ldots, K$, resulting in the matrix equation

$$
\tilde{\boldsymbol{Z}} \tilde{\boldsymbol{P}}=\tilde{\boldsymbol{F}}
$$

where $\quad \tilde{Z}_{m n}^{i j}=\left\langle\tilde{T}_{m}^{i}(\boldsymbol{\rho}), \tilde{\mathcal{G}}_{n}^{j}\left(\boldsymbol{\rho}_{l_{1}^{j}} l_{2}^{j}\right)\right\rangle, \quad$ and $\quad \tilde{F}_{m}^{i}=\left\langle\tilde{T}_{m}^{i}(\boldsymbol{\rho})\right.$, $\left.-\tilde{F}^{\text {inc }}(\boldsymbol{\rho})\right\rangle$. The interaction matrix $\tilde{Z}$ is essentially sparse. Because $\omega$ is assumed to lie within the EC band gap, $\mathcal{G}_{n}^{j}\left(\boldsymbol{\rho}_{l_{1}^{j} l_{2}^{j}}\right)$ decays exponentially with $\left|\boldsymbol{\rho}_{l_{1}^{j} l_{2}^{j}}\right|$. Therefore, each and every replaced cylinder only interacts with its near neighbors, thereby rendering vanishingly small all entries of $\tilde{Z}$ describing 
interactions between sufficiently separated replaced cylinders. Moreover, as shown in [2], such sparse matrix equations can be solved rapidly using multifrontal methods [4].

\section{Fast Calculation of All EC GFs}

No closed-form expressions exist for the EC GF $\tilde{\mathcal{G}}_{n}^{j}\left(\boldsymbol{\rho}_{l_{1}^{j} l_{2}^{j}}\right)$. Fortunately, its numerical calculation is aided by two facts. First, because the EC is periodic, $\tilde{\mathcal{G}}_{n}^{j}\left(\boldsymbol{\rho}_{l_{1}^{j} l_{2}^{j}}\right)$ does not depend on the exact location of irregular cylindrical element $j$. Second, $\tilde{\mathcal{G}}_{n}^{j}(\boldsymbol{\rho})$ decays exponentially with distance. As a consequence, $\tilde{\mathcal{G}}_{n}^{j}(\boldsymbol{\rho})$ can be evaluated using the generalized free-space MST scheme by considering a sufficiently large finite EC of $N_{c}=\left(2 N_{b}+1\right)^{2}$ cylindrical elements, which is assumed to be centered about the spatial origin $\boldsymbol{\rho}=\mathbf{0}$ and which is excited by a Huygens source with components $\tilde{\mathcal{P}}_{1}^{j}(\boldsymbol{\rho})=\left(1 / 2 \pi \tilde{r}_{j}\right) e^{\jmath n \phi} \delta\left(\rho-\tilde{r}_{j}\right) \hat{\boldsymbol{z}}$ and $\tilde{\mathcal{Q}}_{1}^{j}(\boldsymbol{\rho})=$ $\sum_{p=-K}^{K}\left(\mathcal{Y}_{p n}^{j} / 2 \pi \tilde{r}_{j}\right) e^{\jmath p \phi} \delta\left(\rho-\tilde{r}_{j}\right) \hat{\boldsymbol{\phi}}$. In [2], it was shown that matrix (23) can be solved efficiently by using an iterative, preconditioned, and fast Fourier transform-accelerated method.

At this point, it is important to note that it is not necessary to solve a new dense linear system (23) for every EC GF $\tilde{\mathcal{G}}_{n}^{j}(\boldsymbol{\rho}), j=1, \ldots, N_{r}$. Define $\tilde{\mathcal{G}}_{n}^{P}\left(\boldsymbol{\rho}, \tilde{r}_{j}\right)$ as the EC GF for a Huygens source with distributed components $\mathcal{P}_{1}^{j}(\boldsymbol{\rho})=$ $\left(1 / 2 \pi \tilde{r}_{j}\right) e^{\jmath n \phi} \delta\left(\rho-\tilde{r}_{j}\right) \hat{z}$ and $\mathcal{Q}_{1}^{j}(\boldsymbol{\rho})=0 \hat{\boldsymbol{\phi}}$ radiating in the defectless EC. Likewise, define $\tilde{\mathcal{G}}_{n}^{Q}\left(\boldsymbol{\rho}, \tilde{r}_{j}\right)$ as the EC GF for a Huygens source with distributed components $\mathcal{P}_{1}^{j}(\boldsymbol{\rho})=0 \hat{\boldsymbol{z}}$ and $\mathcal{Q}_{1}^{j}(\boldsymbol{\rho})=\left(1 / 2 \pi \tilde{r}_{j}\right) e^{\jmath n \phi} \delta\left(\rho-\tilde{r}_{j}\right) \hat{\boldsymbol{\phi}}$ radiating in the defectless EC. Then, it follows that

$$
\tilde{\mathcal{G}}_{n}^{j}(\boldsymbol{\rho})=\tilde{\mathcal{G}}_{n}^{P}\left(\boldsymbol{\rho}, \tilde{r}_{j}\right)+\sum_{p=-K}^{K} \mathcal{Y}_{p n}^{j} \mathcal{G}_{p}^{Q}\left(\boldsymbol{\rho}, \tilde{r}_{j}\right) .
$$

According to (38), a dense linear system (23) of equations has to be solved for every different radius $\tilde{r}_{j}$. However, it will be shown here that it is sufficient to calculate only $\tilde{\mathcal{G}}_{n}^{P}(\boldsymbol{\rho}, r)$ and $\tilde{\mathcal{G}}_{n}^{Q}(\boldsymbol{\rho}, r)$.

Let $F_{m n}^{j}\left(l_{1}, l_{2}\right)$ denote the element of the excitation vector $\boldsymbol{F}$ in (23) by using the $m$ th harmonic testing function $T_{m}^{i}(\boldsymbol{\rho})$ on the incident fields produced by the Huygens sources on the cylindrical surface with radius $r$ centered about $\rho_{l_{1} l_{2}}^{c}$. For the calculation of $\tilde{G}_{n}^{P}\left(\boldsymbol{\rho}, \tilde{r}_{j}\right)$, the excitation can be expressed as in (39), shown at the bottom of the page. It is readily verified that the same excitation vector is obtained for a Huygens source with distributed components

$$
\begin{aligned}
\mathcal{P}_{1}^{j}(\boldsymbol{\rho})= & -\frac{j k_{b}}{4}\left[H_{n}^{(2)}\left(k_{b} \tilde{r}_{j}\right) J_{n}^{\prime}\left(k_{b} r\right)-J_{n}\left(k_{b} \tilde{r}_{j}\right) H_{n}^{(2)^{\prime}}\left(k_{b} r\right)\right] \\
& \times e^{j n \phi} \delta(\rho-r) \hat{\boldsymbol{z}} \\
= & \frac{1}{2 \pi r} P_{n}^{P}\left(\tilde{r}_{j}\right) e^{j n \phi} \delta(\rho-r) \hat{\boldsymbol{z}} \\
\mathcal{Q}_{1}^{j}(\boldsymbol{\rho})= & \frac{\omega \theta_{b}}{4}\left[H_{n}^{(2)}\left(k_{b} \tilde{r}_{j}\right) J_{n}\left(k_{b} r\right)-J_{n}\left(k_{b} \tilde{r}_{j}\right) H_{n}^{(2)}\left(k_{b} r\right)\right] \\
& \times e^{j n \phi} \delta(\rho-r) \hat{\boldsymbol{\phi}} \\
= & \frac{1}{2 \pi r} Q_{n}^{P}\left(\tilde{r}_{j}\right) e^{j n \phi} \delta(\rho-r) \hat{\boldsymbol{\phi}} .
\end{aligned}
$$

From (40) and (41), it follows that

$$
\tilde{\mathcal{G}}_{n}^{P}\left(\boldsymbol{\rho}, \tilde{r}_{j}\right)=P_{n}^{P}\left(\tilde{r}_{j}\right) \tilde{\mathcal{G}}_{n}^{P}(\boldsymbol{\rho}, r)+Q_{n}^{P}\left(\tilde{r}_{j}\right) \tilde{\mathcal{G}}_{n}^{Q}(\boldsymbol{\rho}, r) .
$$

For the calculation of $\tilde{G}_{n}^{P}\left(\boldsymbol{\rho}, \tilde{r}_{j}\right)$, the excitation can be expressed as in (43), shown at the bottom of the page. It is readily verified that the same excitation vector is obtained for a Huygens source with distributed components

$$
\begin{aligned}
\mathcal{P}_{1}^{j}(\boldsymbol{\rho})= & \frac{\omega \kappa_{b}}{4}\left[H_{n}^{(2)^{\prime}}\left(k_{b} \tilde{r}_{j}\right) J_{n}^{\prime}\left(k_{b} r\right)-J_{n}^{\prime}\left(k_{b} \tilde{r}_{j}\right) H_{n}^{(2)^{\prime}}\left(k_{b} r\right)\right] \\
& \times e^{j n \phi} \delta(\rho-r) \hat{\boldsymbol{z}} \\
= & \frac{1}{2 \pi r} P_{n}^{Q}\left(\tilde{r}_{j}\right) e^{j n \phi} \delta(\rho-r) \hat{\boldsymbol{z}} \\
\mathcal{Q}_{1}^{j}(\boldsymbol{\rho})= & -\frac{j k_{b}}{4}\left[H_{n}^{(2)}\left(k_{b} \tilde{r}_{j}\right) J_{n}^{\prime}\left(k_{b} r\right)-J_{n}\left(k_{b} \tilde{r}_{j}\right) H_{n}^{(2)^{\prime}}\left(k_{b} r\right)\right] \\
& \times e^{j n \phi} \delta(\rho-r) \hat{\boldsymbol{\phi}} \\
= & \frac{1}{2 \pi r} Q_{n}^{Q}\left(\tilde{r}_{j}\right) e^{j n \phi} \delta(\rho-r) \hat{\boldsymbol{\phi}} .
\end{aligned}
$$

From (44) and (45), it follows that

$$
\tilde{\mathcal{G}}_{n}^{Q}\left(\boldsymbol{\rho}, \tilde{r}_{j}\right)=P_{n}^{Q}\left(\tilde{r}_{j}\right) \tilde{G}_{n}^{P}(\boldsymbol{\rho}, r)+Q_{n}^{Q}\left(\tilde{r}_{j}\right) \tilde{G}_{n}^{Q}(\boldsymbol{\rho}, r) .
$$

A combination of (38), (42), and (46) shows that all EC GFs are known once $\mathcal{G}_{n}^{P}(\boldsymbol{\rho}, r)$ and $\tilde{\mathcal{G}}_{n}^{Q}(\boldsymbol{\rho}, r)$ are calculated.

$$
F_{m n}^{j}\left(l_{1}, l_{2}\right)= \begin{cases}\frac{\omega \theta_{b}}{4} H_{n}^{(2)}\left(k_{b} \tilde{r}_{j}\right), & \text { if } l_{1}=l_{2}=0 \text { and } m=n \\ 0, & \text { if } l_{1}=l_{2}=0 \text { and } m \neq n \\ \frac{\omega \theta_{b}}{4} J_{n}\left(k_{b} \tilde{r}_{j}\right) H_{n-m}^{(2)}\left(k_{b} \rho_{l_{1} l_{2}}^{c}\right) e^{\jmath(n-m) \phi_{l_{1} l_{2}}^{c},}, & \text { otherwise. }\end{cases}
$$

$$
F_{m n}^{j}\left(l_{1}, l_{2}\right)= \begin{cases}\frac{\jmath k_{b}}{4} H_{n}^{(2)^{\prime}}\left(k_{b} \tilde{r}_{j}\right), & \text { if } l_{1}=l_{2}=0 \text { and } m=n \\ 0, & \text { if } l_{1}=l_{2}=0 \text { and } m \neq n \\ \frac{\jmath k_{b}}{4} J_{n}^{\prime}\left(k_{b} \tilde{r}_{j}\right) H_{n-m}^{(2)}\left(k_{b} \rho_{l_{1} l_{2}}^{c}\right) e^{\jmath(n-m) \phi_{l_{1} l_{2}}^{c},}, & \text { otherwise. }\end{cases}
$$




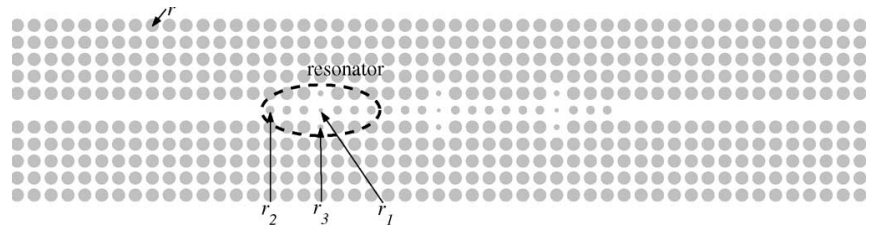

Fig. 4. Structure of the third-order resonant EC filter. The radius of a regular cylinder is $r=0.2 a$. The radii of the irregular cylinders are $r_{1}=0.08 a, r_{2}=$ $0.15 a$, and $r_{3}=0.123 a$.

\section{EXAMPLES}

The examples presented here demonstrate the performance and accuracy of the generalized EC GF MST when applied to the analysis of a wide variety of EC devices including the following:

- devices comprising circular elements with radii and/or constitutive parameters different from those of the background EC (e.g., a third-order Chebyshev bandpass filter or a channel drop filter);

- devices comprising elements that do not snap to the background EC's lattice (e.g., a power divider);

- devices embedded in a triangular lattice EC that supports $\mathrm{TE}_{z}$ fields (e.g., a channel drop filter or a bended waveguide);

- devices that are electromagnetically large (e.g., a multiplexer-demultiplexer);

- devices comprising noncircular elements (e.g., EC waveguide filters loaded with elliptical or starlike cylinders);

- devices comprising plasmonic cylinders (e.g., a plasmonic/dielectric EC waveguide filter).

All calculations are carried out on a $2.4-\mathrm{GHz}$ PC. The multifrontal package used to solve system (37) is UMFPACK Version 4.3 [10].

\section{A. EC-Based Third-Order Bandpass Filter}

Consider the compact third-order resonant filter depicted in Fig. 4 [11]. The filter is embedded in a square (Cartesian) EC comprising homogeneous dielectric circular cylinders with constitutive parameters $\left(\epsilon_{c}, \mu_{c}\right)=\left(11.56 \epsilon_{0}, \mu_{0}\right)$ and radius $r=0.2 a$ that reside in a free-space background, viz. $\left(\epsilon_{b}, \mu_{b}\right)=$ $\left(\epsilon_{0}, \mu_{0}\right)$. The filter comprises three identical abutting resonators in a waveguide created by removing one row of regular EC cylinders, thereby introducing a row of type-I irregular cylinders. Each resonator comprises a symmetric constellation of nine type-II irregular cylinders that differ from those of the background EC only in terms of their radii (Fig. 4). The filter's $\mathrm{TM}_{z}$ transmission spectrum computed using the generalized EC and free-space GF MST is shown in Fig. 5. When using the generalized EC GF MST, the EC is assumed infinite, and exact modal excitations and boundary conditions are imposed; their construction is facilitated by the fact that the EC GF technique permits a straightforward identification of the propagating EC waveguide modes [2]. When using the free-space GF MST, the waveguide is lined by seven rows of cylinders to avoid leakage and truncated by perfectly matched layer-based absorbing boundary conditions to mimic semi-infinite waveguide loads [12]. Divergence in their underlying assumptions notwithstand-

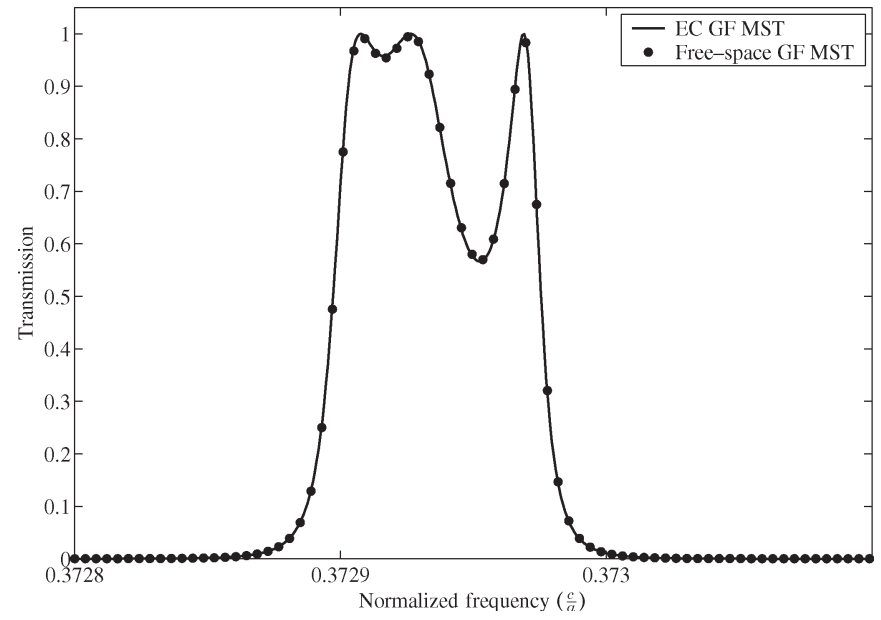

Fig. 5. Transmission spectrum of the third-order resonant EC filter.

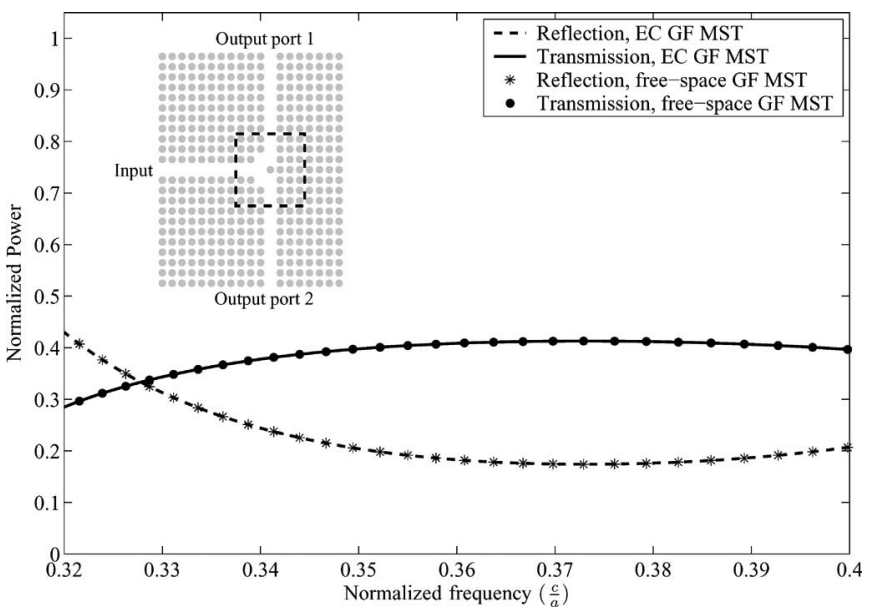

Fig. 6. Structure and transmission spectrum of the EC power divider.

ing, both approaches yield nearly identical transmission spectra. The difference between their CPU requirements, however, is significant. Indeed, application of the generalized EC GF MST with $K=1$ and $N_{b}=20$ requires only 317 unknowns to describe equivalent currents on all irregular (type I + II) cylinders, $5.4 \mathrm{~s}$ to construct EC GFs for type-I and type-II irregular cylinders, $0.05 \mathrm{~s}$ to construct modal excitations and boundary conditions, and $0.05 \mathrm{~s}$ to fill the sparse interaction matrix and solve the resulting system of equations. Hence, the total time to analyze the filter is only $5.5 \mathrm{~s}$ - these CPU times are, just like all that follow, for one frequency. In contrast, application of the free-space GF MST requires 3213 unknowns to describe equivalent currents on all regular and type-II irregular cylinders and $238 \mathrm{~s}$ to fill and solve the resulting system of equations, the bulk of which is consumed by the matrix solution process. Clearly, the generalized EC GF MST is far more efficient at analyzing this structure than its classical counterpart.

\section{B. EC-Based Power Divider}

Next, consider the T-shaped EC power divider depicted in the inset of Fig. 6 [13]. The divider is embedded in the background EC described in Section V-A. The divider is constructed by removing cylinders from the EC, i.e., all irregular cylinders 


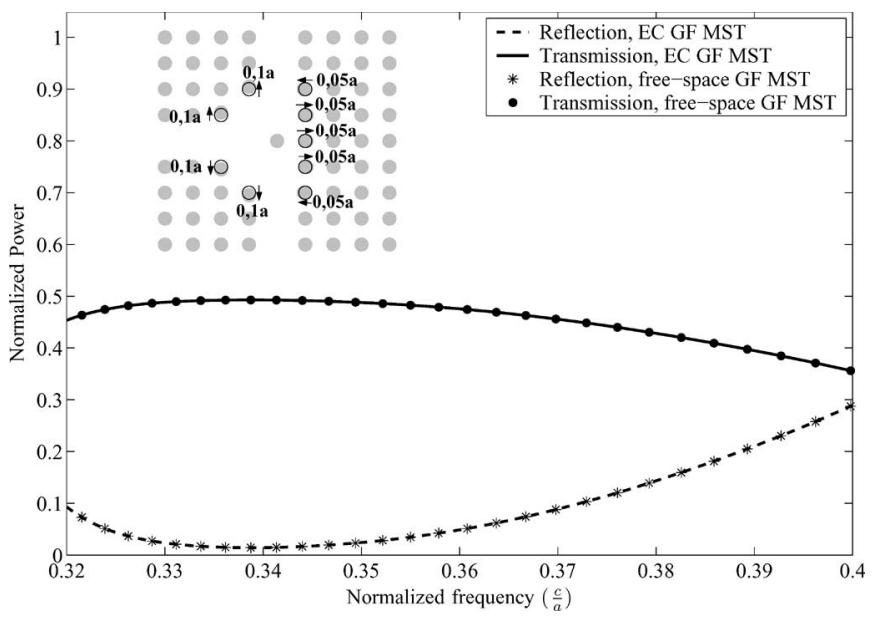

Fig. 7. Structure and transmission spectrum of the adjusted EC power divider.

are of type-I. The divider's $\mathrm{TM}_{z}$ reflection and transmission spectra computed using the generalized EC and free-space GF MSTs are shown in Fig. 6; excellent agreement between both data sets is observed. The divider's properties are far from desirable but can be improved, as was done in [13], by adjusting the constitutive parameters, radii, and/or center positions of the cylinders near the T-junction (type-II irregular cylinders). One such adjusted design, which was obtained by moving the cylinders in the marked square in Fig. 6 without changing their constitutive parameters or radii, is detailed in the inset of Fig. 7, which shows the transmission spectra of the adjusted divider calculated with the generalized EC and free-space GF MST. Again, excellent agreement between both data sets is observed. Application of the generalized EC GF MST with $K=2$ and $N_{b}=23$ requires only 523 unknowns to describe equivalent currents on irregular (type I + II) cylinders, $30 \mathrm{~s}$ to construct the type-I and type-II EC GFs, $0.14 \mathrm{~s}$ to construct modal excitations and boundary conditions, and $0.5 \mathrm{~s}$ to fill the sparse interaction matrix and solve the resulting system of equations. The total CPU time is $30.64 \mathrm{~s}$ and is significantly less than that required by the free-space GF MST, which is $134 \mathrm{~s}$. The (deterministic/evolutionary) synthesis of EC devices typically calls for the evaluation of hundreds if not thousands of design candidates; the preceding example demonstrates the potential usefulness of EC GF MST methods for this purpose. Moreover, note that it is only necessary to calculate the EC GFs $\tilde{\mathcal{G}}^{P}(\boldsymbol{\rho}, r)$ and $\tilde{\mathcal{G}}^{Q}(\boldsymbol{\rho}, r)$ at the first iteration step. Hence, from the second iteration step on, the evaluation of a design candidate would require less than $1 \mathrm{~s}$ !

\section{Channel Drop Filters}

Channel drop filters are key components of many wavelength-division multiplexing communication systems [14]-[18]. Not surprisingly, in recent years, a host of EC-based channel drop filter designs has been proposed; two distinct layouts are analyzed here.

The first filter is depicted in Fig. 8 [16]. This filter is embedded in the EC described in Section V-A. All EC waveguides are formed by removing rows of cylinders. The so-called bus waveguide-through which the EC device is excited-is

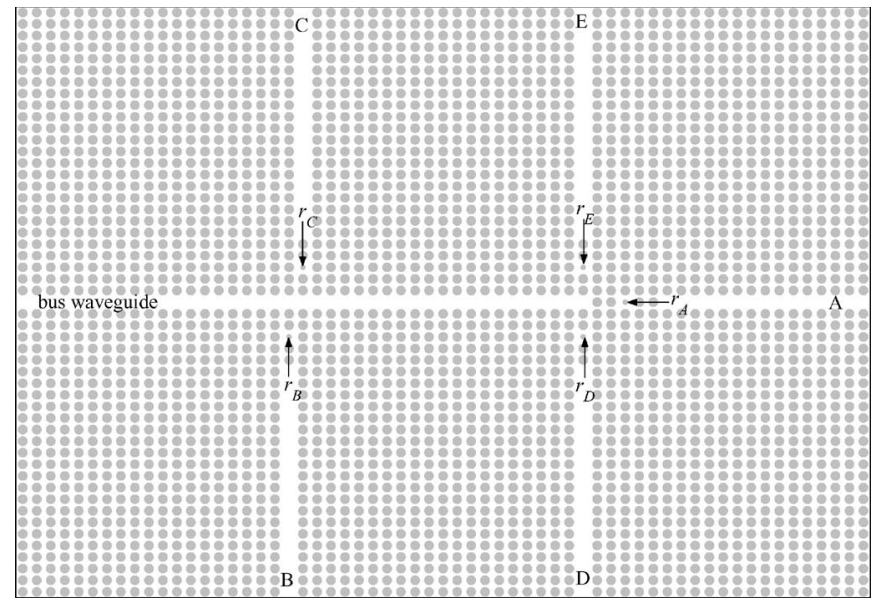

Fig. 8. Structure of the five-channel drop filter. The radii of the irregular cylinders are $r_{A}=0.1 a, r_{B}=0.08 a, r_{C}=0.065 a, r_{D}=0.05 a$, and $r_{E}=0$.

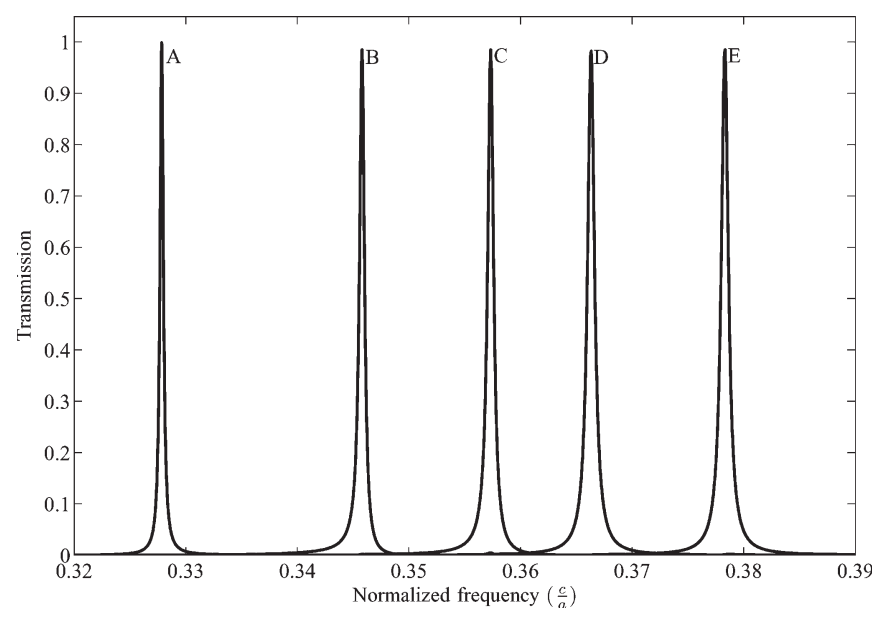

Fig. 9. Transmission spectrum of the five-channel drop filter.

connected to five output waveguides by distinct resonators. Each resonator comprises five cylinders. The four outermost cylinders in each resonator belong to the background EC; the center cylinder in each resonator is irregular as it has a radius that differs from those in the EC-specific radii are detailed in Fig. 8. Fig. 9 shows the filter's transmission spectrum calculated via the generalized EC GF MST. This spectrum agrees well with the finite-difference time-domain method result obtained in [16]. With $K=1$, this example calls for 408 equivalent currents. The time to calculate the EC GF and to construct the modal excitations/boundary conditions is the same as that in Section V-A. Filling and solving the sparse linear system of equations take $0.3 \mathrm{~s}$. The total time to analyze the device for one frequency therefore is only $5.75 \mathrm{~s}$. Analysis of the EC device via the free-space GF MST would require more than 9000 unknowns to describe equivalent currents. Needless to say, the CPU time required to analyze the device via the freespace GF MST would be several orders of magnitude larger than that required by the EC GF MST.

The second filter considered is depicted in the inset of Fig. 10 [15]. This filter is embedded in a triangular lattice EC of air holes $\left(\left(\epsilon_{c}, \mu_{c}\right)=\left(\epsilon_{0}, \mu_{0}\right)\right)$ of radius $r=0.29 a$ residing in a homogeneous background with constitutive parameters 


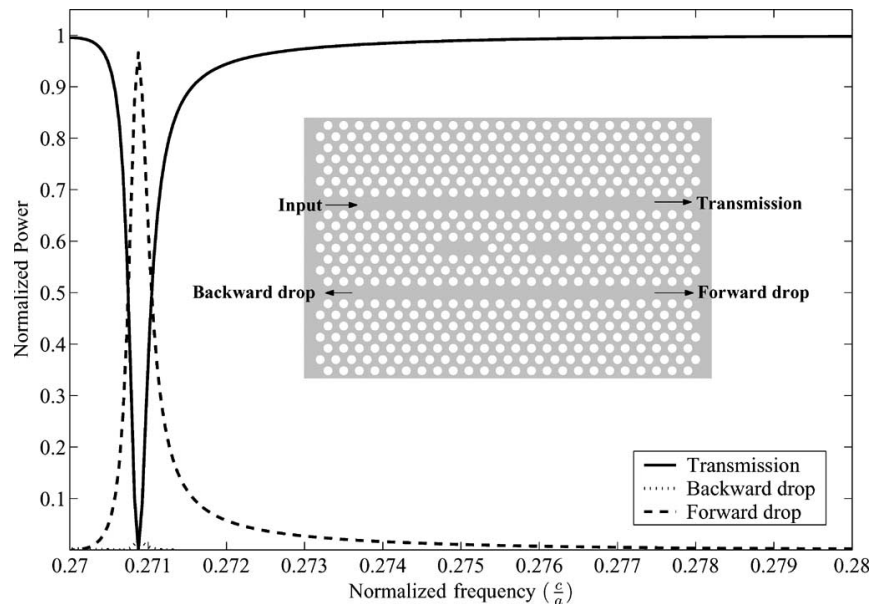

Fig. 10. Structure and transmission spectrum of the channel drop filter.

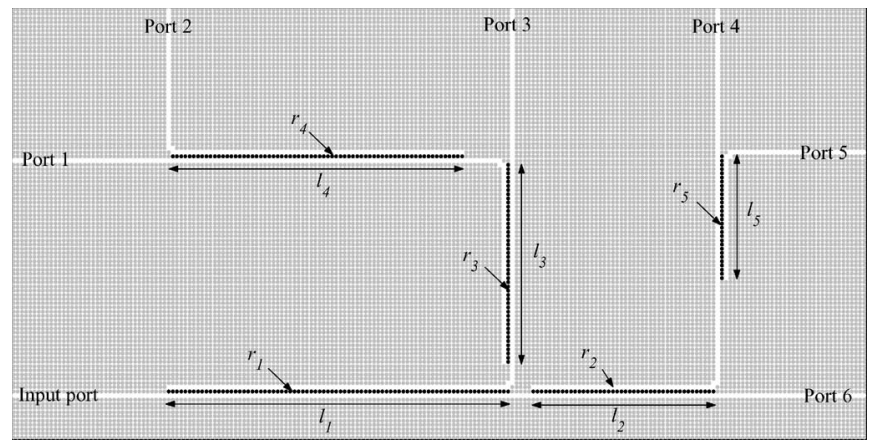

Fig. 11. Structure of the large multiplexer-demultiplexer. The radius of a regular cylinder is $r=0.2 a$. The radii of the irregular cylinders are $r_{1}=$ $0.16 a, r_{2}=0.18 a, r_{3}=0.22 a, r_{4}=0.17 a$, and $r_{5}=0.14 a$. The lengths of the coupling regions are $l_{1}=82 a, l_{2}=44 a, l_{3}=48 a, l_{4}=70 a$, and $l_{5}=30 a$

$\left(\epsilon_{b}, \mu_{b}\right)=\left(7.6176 \epsilon_{0}, \mu_{0}\right)$. The filter comprises two identical EC waveguides that couple through identical resonant cavities. The waveguides and cavities are obtained by filling holes with background material, i.e., the structure only contains type-I irregularities. The cavities each span three hole locations and are separated by two holes. Fig. 10 shows the transmission spectrum calculated with the generalized EC GF MST. Excellent agreement is observed with the spectrum calculated with the finite-difference time-domain technique given in [15]. With $K=2$ and $N_{b}=20$, the generalized EC GF MST requires only 534 unknowns to model equivalent currents on the type-I irregular cylinders, $36 \mathrm{~s}$ to calculate the EC GF, $0.13 \mathrm{~s}$ to construct modal excitations and boundary conditions, and $0.65 \mathrm{~s}$ to fill and solve the resulting system of equations. Hence, the total time to analyze the device is only $30.78 \mathrm{~s}$.

\section{Multiplexer-Demultiplexer Based on EC Waveguide Couplers}

The ability of the generalized EC GF MST to efficiently analyze large EC devices is demonstrated via its application to the six-channel multiplexer-demultiplexer depicted in Fig. 11. The multiplexer-demultiplexer is embedded in the EC described in Section V-A. The device comprises several EC waveguides that are formed by removing a row of type-I

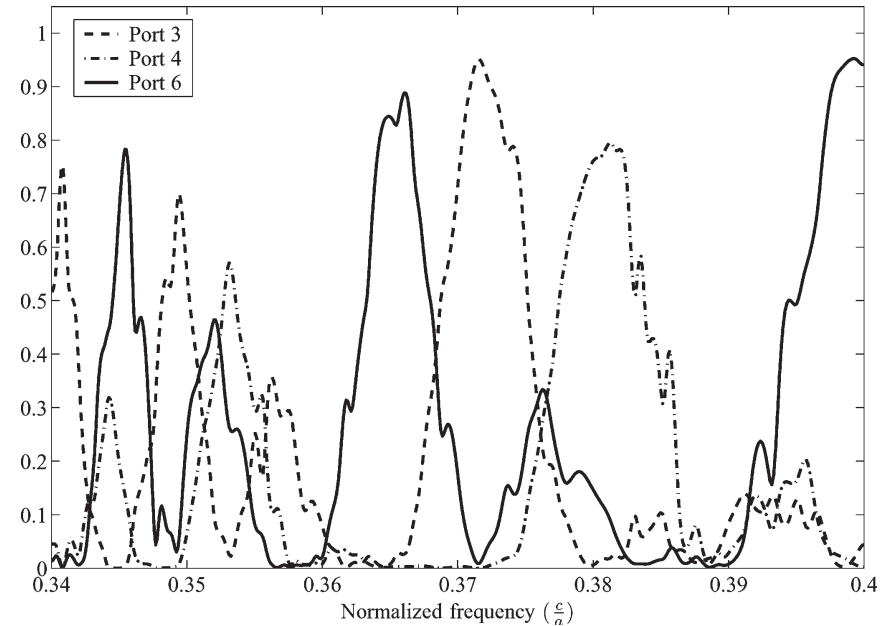

Fig. 12. Transmission spectrum of the multiplexer-demultiplexer.

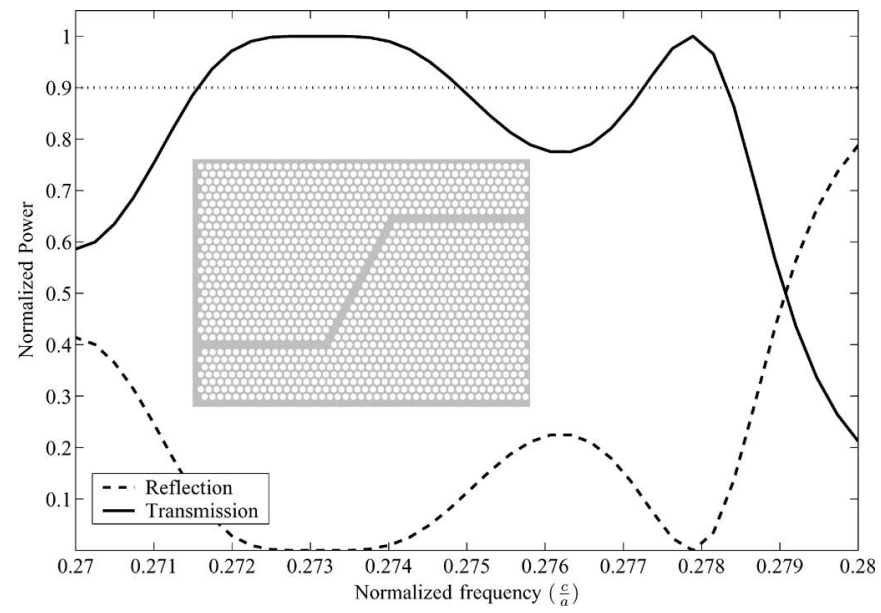

Fig. 13. Structure and transmission spectrum of the EC waveguide with two nonoptimized $60^{\circ}$ bends.

cylinders. In five coupling regions, these waveguides are separated only by one row of type-II cylinders. The waveguide coupling lengths are $l_{1}=82 a, l_{2}=44 a, l_{3}=48 a, l_{4}=70 a$, and $l_{5}=30 a$. The radii of the type-II cylinders that separate the waveguides are $r_{1}=0.16 a, r_{2}=0.18 a, r_{3}=0.22 a$, $r_{4}=0.17 a$, and $r_{5}=0.14 a$. Their permittivity equals that of the EC background cylinders. Fig. 12 shows the transmission spectrum for three of the six output ports. With $K=1$, analysis of this multiplexer-demultiplexer calls for 3349 equivalent currents. The time to calculate the EC GF and to construct the modal excitations/boundary conditions is the same as in Section V-A. Filling and solving the sparse linear system of equations take $3 \mathrm{~s}$. Hence, the total time to analyze the multiplexer-demultiplexer is only 8.4 s. For comparison, when modeled with the conventional free-space GF MST, this large multiplexer-demultiplexer would require more than 60000 equivalent currents.

\section{E. Waveguide Bends}

The ability to efficiently guide light along bend waveguides is critical to many photonics applications. In recent years, 


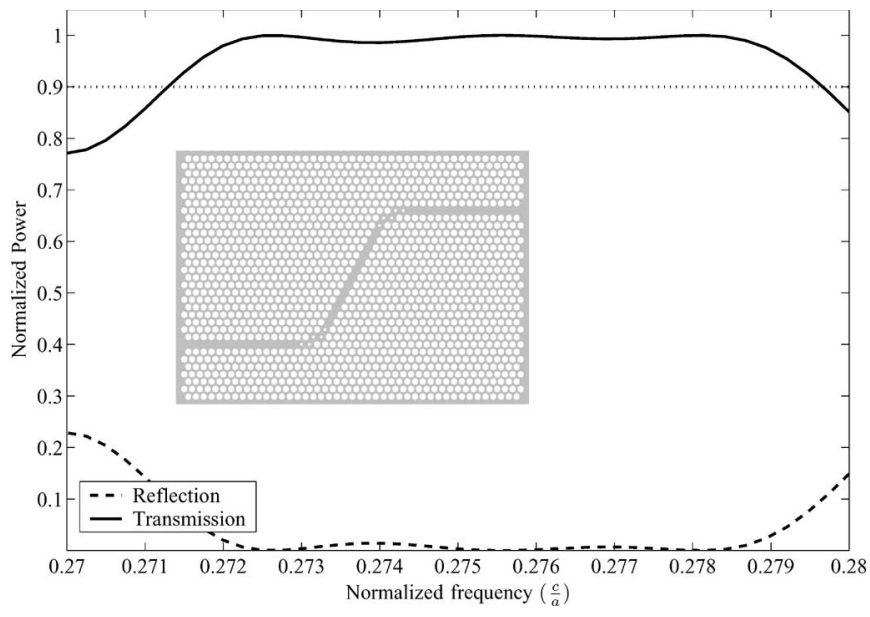

Fig. 14. Structure and transmission spectrum of the EC waveguide with two optimized $60^{\circ}$ bends following [19].

many different EC bend waveguide configurations have been proposed; two specific configurations are analyzed here.

First, consider the crude bend waveguide depicted in the inset of Fig. 13 [19]. The waveguide is embedded in the same triangular lattice EC considered in the second example of Section V-C. The bend waveguide is constructed exclusively by filling EC voids; hence, it comprises only type-I irregularities. The waveguide's $\mathrm{TE}_{z}$ reflection and transmission spectra, which were calculated with the generalized EC GF MST, are shown in Fig. 13. Unfortunately, the power transmission coefficient only exceeds 0.9 in frequencies ranging from $f=$ $0.2716(c / a)$ to $f=0.275(c / a)$ and $f=0.2773(c / a)$ to $f=$ $0.2783(c / a)$. This example required 302 unknowns to model equivalent currents on type-I irregular cylinders, $36 \mathrm{~s}$ to calculate the EC GF, $0.13 \mathrm{~s}$ to construct modal excitations and boundary conditions, and $0.15 \mathrm{~s}$ to fill and solve the linear system of equations.

Second, consider the optimized bend waveguide depicted in the inset of Fig. 14 [19]. The waveguide portion of the optimized structure is identical to that of the previously described crude bend waveguide; the bend regions, however, differ as type-II irregularities in the form of extra air holes with a radius of $0.14 a$ are added. The optimized waveguide's $\mathrm{TE}_{z}$ reflection and transmission spectra computed using the EC GF MST are shown in Fig. 14. The optimized waveguide achieves continuous high transmission from $f=0.2714(c / a)$ up to $f=0.2795(c / a)$. The (extra) computational cost associated with the analysis of this optimized design is very low. Indeed, assuming that the EC GF $\tilde{\mathcal{G}}^{P}(\boldsymbol{\rho}, r)$ and $\tilde{\mathcal{G}}^{Q}(\boldsymbol{\rho}, r)$ and the modal excitations/boundary conditions computed while analyzing the nonoptimized structure remain available, analysis of the optimized design requires only $0.2 \mathrm{~s}$ to fill and store the sparse system of equations for the optimized design.

\section{F. ECS Comprising Noncircular Cylindrical Elements}

All previous examples concerned EC devices comprising circular cylinders for which the matrices $\mathcal{Y}^{j}$ in (19) and (34) are known analytically. Many practical EC devices, however, com-

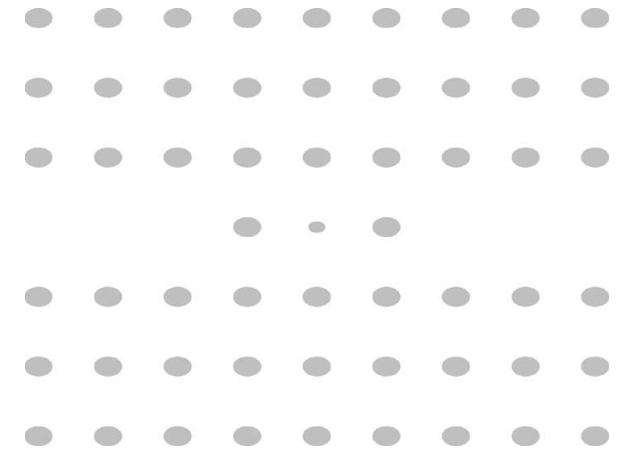

Fig. 15. EC filter with elliptical dielectric cylinders.

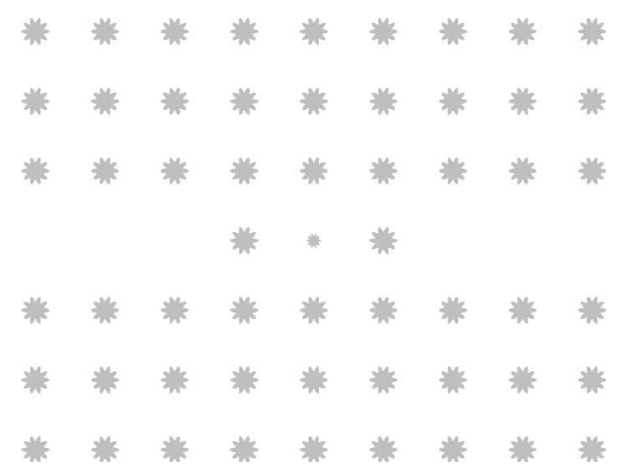

Fig. 16. EC filter with starlike dielectric cylinders.

prise noncircular elements; deviations from the circular shape may be intended or due to manufacturing imperfections. The ability of the generalized EC GF MST to efficiently simulate such EC devices is demonstrated via its application to the EC waveguide filters depicted in Figs. 15 and 16. Both filters are embedded in a square (Cartesian) EC comprising homogeneous noncircular dielectric cylindrical elements with constitutive parameters $\left(\epsilon_{c}, \mu_{c}\right)=\left(11.56 \epsilon_{0}, \mu_{0}\right)$ and comprise a waveguide loaded with three cylindrical elements. The two outermost are identical to those of the EC background. The center element has the same shape but is smaller. The elements in the filter shown in Fig. 15 are ellipses [20]. The major axes of the elliptical cylinders are aligned with the waveguide channel. The large (small) ellipse's major and minor axes measure $0.4 a(0.24 a)$ and $0.28 a(0.16 a)$, respectively. The cylindrical elements in the filter shown in Fig. 15 are starlike with angle-dependent radius given by

$$
r(\theta)=r_{1}+r_{2} \cos (m \theta), \quad \theta=0, \ldots, 2 \pi .
$$

For the large (small) cylindrical elements, $r_{1}=0.16\left(r_{1}=\right.$ $0.08), r_{2}=0.04\left(r_{2}=0.02\right)$, and $m=10(m=10)$. Fig. 17 shows the transmission spectrum of both EC filters calculated with the generalized EC GF MST. For both examples, the matrices $\mathcal{Y}^{j}$ were calculated numerically with a simple volume integral-equation-based scheme [21]. Application of the generalized EC GF MST with $K=2$ and $N_{b}=20$ requires only 192 unknowns to describe the fields in the channel and uses $9.34 \mathrm{~s}$ to construct the type-I and type-II EC GFs, $0.14 \mathrm{~s}$ to construct 


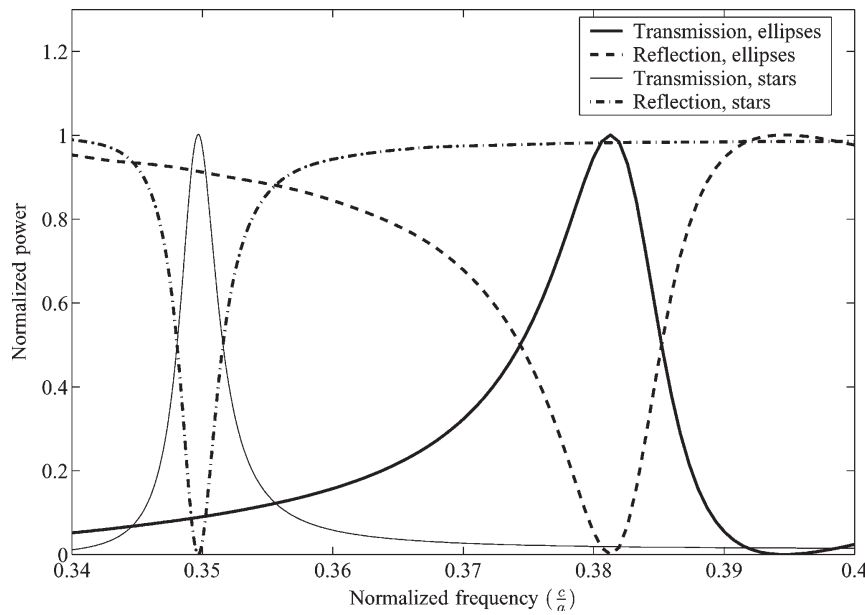

Fig. 17. Transmission spectrum of the EC filters with elliptical and starlike dielectric cylinders.

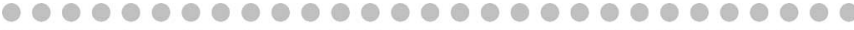
으응 - 000000000000000000000000000

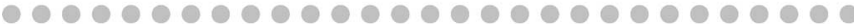
Plasmonic cylinders, 0 permittivity $\varepsilon_{1}$

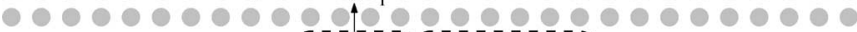
ó

-

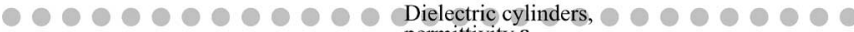
00000000000 permittivity $\varepsilon_{2} 0000000000$

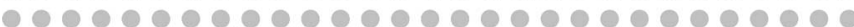
○ ৩

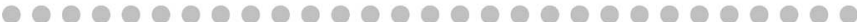
-

Fig. 18. Structure of the plasmonic/dielectric EC filter. The radius of all cylinders is $r=0.2 a$. The permittivity of the plasmonic cylinders is $\epsilon_{1}=$ $\epsilon_{0}\left[1-\left(f_{p} / f\right)^{2}\right]$, with $f_{p}=0.662(c / a)$. The permittivity of the irregular dielectric cylinders is $\epsilon_{2}=4.9 \epsilon_{0}$.

modal excitations and boundary conditions, and $0.03 \mathrm{~s}$ to fill and solve the sparse system of equations.

\section{G. ECS Comprising Plasmonic Elements}

In recent years, the development of optical devices that incorporate plasmonic materials has attracted significant attention [22]. In this section, it will be shown that a bandpass filter can be designed by loading an EC waveguide with plasmonic and dielectric cylinders. Consider the plasmonic/dielectric EC device depicted in Fig. 18. The device is embedded within the EC described in Section V-A. The EC waveguide is loaded with four plasmonic cylinders followed by six dielectric cylinders. The radius and permittivity of the plasmonic cylinders are $r_{1}=$ $0.2 a$ and $\epsilon_{1}=\epsilon_{0}\left[1-(f p / f)^{2}\right]$, where the plasmonic frequency $f_{p}=0.662(c / a)$. The radius and permittivity of the irregular dielectric cylinders are $r_{2}=0.2 a$ and $\epsilon_{2}=4.9 \epsilon_{0}$, respectively. Fig. 19 shows the transmission spectrum calculated with the generalized EC GF MST. Within the frequency range shown, the four plasmonic cylinders act as a high-pass filter, while the six irregular dielectric cylinders behave as a low-pass filter. Consequently, the combined structure behaves as a bandpass filter. With $K=1$ and $N_{b}=20$, this device calls for 152 equivalent currents. The time to calculate the EC GF and to construct

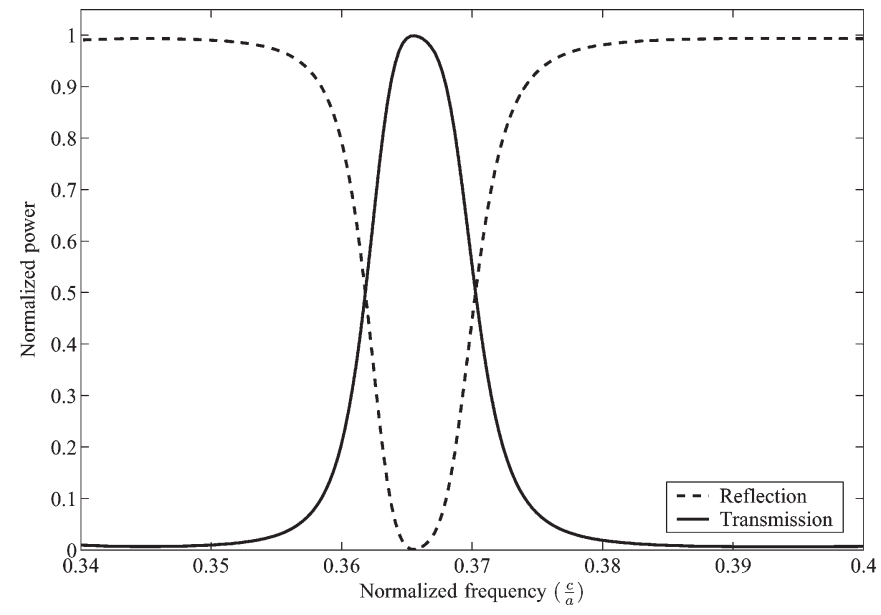

Fig. 19. Transmission spectrum of the plasmonic/dielectric EC filter.

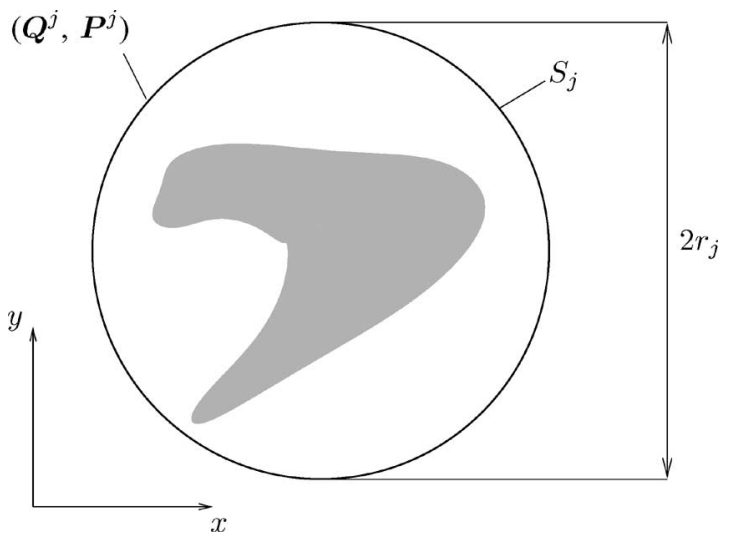

Fig. 20. Relation between $\mathcal{Y}^{j}$ and $\mathcal{S}^{j}$

the modal excitations/boundary conditions is the same as that in Section V-A. Filling and solving the sparse linear system of equations take only $0.03 \mathrm{~s}$.

\section{CONCLUSION}

A generalized EC GF MST enabling the characterization of EC devices obtained by replacing cylindrical elements from an infinite doubly periodic and defectless EC by nonconforming ones was presented. Both EC background and nonconforming cylinders can be of arbitrary shape and composition. When combined with a volume integral-equation (or finite-elements)based scheme to construct scattering matrices of nonconforming cylindrical elements, the generalized EC GF MST can handle a much larger class of EC devices than the original EC GF MST presented in [2]. The versatility of the generalized scheme was demonstrated via its application to several practical examples. An extension of the generalized EC GF MST to the characterization of 3-D EC slab devices, i.e., slab waveguides strategically loaded with 2-D ECs, is under study. Very recently, a 3-D version of the free-space GF MST was published [23]. It is anticipated that a combination of the latter scheme with fast matrix-vector multiplication algorithms will permit the computationally efficient calculation of 3-D EC GFs. This, in turn, will permit the extension of the generalized EC GF MST to 


$$
F^{\text {inc }}(\boldsymbol{\rho})= \begin{cases}-\frac{\omega \theta_{b}}{4} \sum_{n=-K}^{K}\left[H_{n}^{(2)}\left(k_{b} r_{j}\right) P_{n}^{j}+\jmath \sqrt{\frac{\kappa_{b}}{\theta_{b}}} H_{n}^{(2))^{\prime}}\left(k_{b} r_{j}\right) Q_{n}^{j}\right] J_{n}\left(k_{b} \rho_{j}\right) e^{j n \phi_{j},}, & \text { if } \rho_{j}<r_{j} \\ -\frac{\omega \theta_{b}}{4} \sum_{n=-K}^{K}\left[J_{n}\left(k_{b} r_{j}\right) P_{n}^{j}+\jmath \sqrt{\frac{\kappa_{b}}{\theta_{b}}} J_{n}^{\prime}\left(k_{b} r_{j}\right) Q_{n}^{j}\right] H_{n}^{(2)}\left(k_{b} \rho_{j}\right) e^{j n \phi_{j}}, & \text { if } \rho_{j}>r_{j}\end{cases}
$$

3-D and the subsequent computationally efficient characterization of 3-D EC slab devices.

\section{APPENDIX}

This Appendix details the relationship between the scattering matrix $\mathcal{S}^{j}$ and the matrix $\mathcal{Y}^{j}$, as used in (19) and (34), of a cylindrical element embedded in a homogeneous medium with constitutive parameters $\left(\epsilon_{b}, \mu_{b}\right)$. Consider the cylindrical element depicted in Fig. 20. The scattering matrix $\mathcal{S}^{j}$ relates scattered fields to incident fields cast in terms of Bessel/Hankel functions, i.e., if the incident and scattered fields on $S_{j}$ are expressed as

$$
\begin{gathered}
\left.F^{\mathrm{inc}}(\boldsymbol{\rho})\right|_{\boldsymbol{\rho} \in S_{j}}=-\frac{\omega \theta_{b}}{4} \sum_{m=-K}^{K} A_{n}^{j} J_{n}\left(k_{b} r_{j}\right) e^{j n \phi_{j}} \\
\left.F^{\mathrm{sca}, \mathrm{j}}(\boldsymbol{\rho})\right|_{\boldsymbol{\rho} \in S_{j}}=-\frac{\omega \theta_{b}}{4} \sum_{m=-K}^{K} B_{n}^{j} H_{n}^{(2)}\left(k_{b} r_{j}\right) e^{j n \phi_{j}}
\end{gathered}
$$

then

$$
B_{n}^{j}=\sum_{p=-K}^{K} \mathcal{S}_{n p}^{j} A_{p}^{j} \quad \text { or } \quad \boldsymbol{B}^{j}=\mathcal{S}^{j} \boldsymbol{A}^{j} .
$$

The scattering matrix can be found with several numerical schemes, such as, e.g., a volume integral-equation-based scheme or a finite-element-based scheme. For circular elements, the scattering matrix is known analytically.

According to (16) and (31), the unknown currents $P_{n}^{j}$ and $Q_{n}^{j}$ should relate in such a way that when radiating around cylindrical element $j$, they generate zero fields outside the surface $S_{j}$. This relationship can be found easily via the scattering matrix of the cylindrical element. First, calculate the field that is radiated by arbitrary $P_{n}^{j}$ and $Q_{n}^{j}$ in the homogeneous medium with constitutive parameters $\left(\epsilon_{b}, \mu_{b}\right)$. By definition, the field incident on the cylindrical element is as in (A.4), shown at the top of the page. Second, define the diagonal matrices $\boldsymbol{D}_{1}, \boldsymbol{D}_{2}$, $\boldsymbol{D}_{3}$, and $\boldsymbol{D}_{4}$ with entries

$$
\begin{aligned}
& {\left[\boldsymbol{D}_{1}\right]_{n n}=H_{n}^{(2)}\left(k_{b} r_{j}\right)} \\
& {\left[\boldsymbol{D}_{2}\right]_{n n}=\sqrt{\frac{\kappa_{b}}{\theta_{b}}} H_{n}^{(2)^{\prime}}\left(k_{b} r_{j}\right)} \\
& {\left[\boldsymbol{D}_{3}\right]_{n n}=J_{n}\left(k_{b} r_{j}\right)} \\
& {\left[\boldsymbol{D}_{4}\right]_{n n}=\jmath \sqrt{\frac{\kappa_{b}}{\theta_{b}}} J_{n}^{\prime}\left(k_{b} r_{j}\right) .}
\end{aligned}
$$

Third, combine (16), (31), and (A.1) -(A.4) to find the relationship between the unknown $P_{n}^{j}$ and $Q_{n}^{j}$

$$
\boldsymbol{Q}=-\left[\mathcal{S}^{j} \boldsymbol{D}_{2}+\boldsymbol{D}_{4}\right]^{-1}\left[\mathcal{S}^{j} \boldsymbol{D}_{1}+\boldsymbol{D}_{3}\right] \boldsymbol{P}
$$

or

$$
\mathcal{Y}^{j}=-\left[\mathcal{S}^{j} \boldsymbol{D}_{2}+\boldsymbol{D}_{4}\right]^{-1}\left[\mathcal{S}^{j} \boldsymbol{D}_{1}+\boldsymbol{D}_{3}\right]
$$

\section{ACKNOWLEDGMENT}

D. Pissoort would like to thank the Fund for Scientific Research-Flanders (Belgium) (FWO-Vlaanderen) for a postdoctoral fellowship.

\section{REFERENCES}

[1] D. Pissoort, B. Denecker, P. Bienstman, F. Olyslager, and D. De Zutter, "Comparative study of three methods for the simulation of twodimensional photonic crystals," J. Opt. Soc. Amer. A, Opt. Image Sci., vol. 21, no. 11, pp. 2186-2195, Nov. 2004.

[2] D. Pissoort, E. Michielssen, F. Olyslager, and D. De Zutter, "Fast analysis of $2 \mathrm{D}$ electromagnetic crystal structures using a periodic Green function approach," J. Lightw. Technol., vol. 23, no. 7, pp. 2294-2308, Jul. 2005.

[3] G. Tayeb and D. Maystre, "Rigorous theoretical study of finite-size twodimensional photonic crystals doped by microcavities," J. Opt. Soc. Amer. A, Opt. Image Sci., vol. 14, no. 12, pp. 3323-3332, Dec. 1997.

[4] I. S. Duff and J. K. Reid, "The multifrontal solution of unsymmetric sets of linear equations," SIAM J. Sci. Comput., vol. 5, no. 3, pp. 633-641, 1984.

[5] D. Pissoort, E. Michielssen, F. Olyslager, and D. De Zutter, "A rank revealing preconditioner for the fast integral equation based characterization of electromagnetic crystal devices," Microw. Opt. Technol. Lett., vol. 48, no. 4, pp. 783-789, Apr. 2006.

[6] T. Fujisawa and M. Koshiba, "Finite-element modeling of nonlinear Mach-Zehnder interferometers based on photonic-crystal waveguides for all-optical signal processing," J. Lightw. Technol., vol. 24, no. 1, pp. 617623, Jan. 2006.

[7] S. K. Chang and K. K. Mei, "Application of unimoment method to electromagnetic scattering of dielectric cylinders," IEEE Trans. Antennas Propag., vol. AP-24, no. 1, pp. 35-42, Jan. 1976.

[8] K. K. Mei, "Unimoment method for electromagnetic-wave scattering," J. Electromagn. Waves Appl., vol. 1, no. 3, pp. 201-222, 1987.

[9] R. E. Collin, Field Theory of Guided Waves. New York: IEEE Press, 1991.

[10] [Online]. Available: http://www.cise.ufl.edu/davis/

[11] D. Park, S. Kim, I. Park, and H. Lim, "Higher order optical resonant filters based on coupled defect resonators in photonic crystals," J. Lightw. Technol., vol. 23, no. 5, pp. 1923-1928, May 2005.

[12] D. Pissoort and F. Olyslager, "Termination of periodic waveguides by PMLs in time-harmonic integral equation like techniques," IEEE Antennas Wireless Propag. Lett., vol. 2, pp. 281-284, 2003.

[13] J. Smajic, C. Hafner, and D. Erni, "Optimization of photonic crystal structures," J. Opt. Soc. Amer. A, Opt. Image Sci., vol. 21, no. 11, pp. 2223 2232, Nov. 2004.

[14] S. Fan, P. R. Villeneuve, and J. D. Joannopoulos, "Channel drop filters in photonic crystals," Opt. Express, vol. 3, no. 1, pp. 4-11, Jul. 1998.

[15] B. K. Min, J. E. Kim, and H. Y. Park, "Channel drop filters using resonant tunneling processes in two-dimensional triangular lattice photonic crystal slabs," Opt. Commun., vol. 237, no. 1-3, pp. 59-63, 2004. 
[16] S. Kim, I. Park, and H. Lim, "Highly efficient photonic crystal-based multichannel drop filters of three-port system with reflection feedback," Opt. Express, vol. 12, no. 22, pp. 5518-5525, Nov. 2004.

[17] E. Drouard, H. T. Hattori, C. Grillet, A. Kazmierczak, X. Letartre, P. Rojo-Romeo, and P. Viktotovich, "Directional channel-drop filter based on a slow bloch mode photonic crystal waveguide section," Opt. Express, vol. 13, no. 8, pp. 3037-3048, Apr. 2005.

[18] Z. Zhang and M. Qiu, "Compact in-plane channel drop filter design using a single cavity with two degenerate modes in 2D photonic crystal slabs," Opt. Express, vol. 13, no. 7, pp. 2596-2604, Apr. 2005.

[19] A. Chutinan, M. Okano, and S. Noda, "Wider bandwidth with high transmission through waveguide bends in two-dimensional photonic crystal slabs," Appl. Phys. Lett., vol. 80, no. 10, pp. 1698-1700, Mar. 2002.

[20] N. J. Florous, K. S. Saitoh, and M. Koshiba, "Modeling of twodimensional photonic crystal resonant cavities incorporating elliptically shaped dielectric cylinders," IEEE Photon. Technol. Lett., vol. 17, no. 11, pp. 2316-2318, Nov. 2005.

[21] A. F. Peterson, S. L. Ray, and R. Mittra, Computational Methods for Electromagnetics, ser. IEEE Press Series on Electromagnetic Waves. New York: IEEE Press, 1998.

[22] S. A. Maier and H. A. Atwater, "Plasmonics: Localization and guiding of electromagnetic energy in metal/dielectric structures," J. Appl. Phys., vol. 98, no. 1, pp. 011101.1-011101.10, Jul. 2005.

[23] S. Boscolo and M. Midrio, "Three-dimensional multiple-scattering technique for the analysis of photonic-crystal slabs," J. Lightw. Technol., vol. 22 , no. 12, pp. 2778-2786, Dec. 2004.

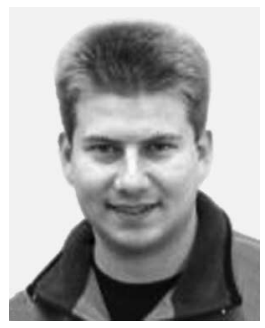

Davy Pissoort was born in 1978. He received the M.S. and Ph.D. degrees in electrical engineering from Ghent University, Ghent, Belgium, in 2001 and 2005, respectively.

From October 2005 to October 2006, he was a Postdoctoral Researcher with the Fund for Scientific Research-Flanders (Belgium) (FWO-Vlaanderen) with the Department of Information Technology, Ghent University. In November 2006, he joined the Eesof-EDA Department, Agilent Technolgies, Ghent, Belgium, as a Research Engineer. His current research interests include the analysis of electromagnetic waveguides and photonic-crystal devices, as well as the development of fast and efficient electromagnetic simulation methods.

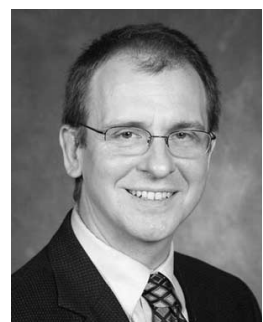

Eric Michielssen (M'95-SM'99-F'02) received the M.S. degree (summa cum laude) in electrical engineering from Katholieke Universiteit Leuven (KUL), Leuven, Belgium, in 1987 and the Ph.D. degree in electrical engineering from the University of Illinois at Urbana-Champaign (UIUC) in 1992.

He was a Research and Teaching Assistant with the Microwaves and Lasers Laboratory, KUL, and the Electrical Communication Laboratory, UIUC, from 1987 to 1988 and from 1988 to 1992 , respectively. He joined the faculty of the Department of Electrical and Computer Engineering, UIUC, as a Visiting Assistant Professor in 1992 and became an Assistant Professor of electrical and computer engineering in 1993 and an Associate Professor in 1998. Since 1995, he has been an Associate Director of the Center for Computational Electromagnetics, UIUC. In 2005, he joined the Radiation Laboratory, Department of Electrical Engineering and Computer Science, University of Michigan, Ann Arbor, as a Professor of electrical engineering and computer science. He is also a Beckman Fellow with the Center for Advanced Studies. He authored and coauthored more than 130 journal papers and book chapters and more than 190 conference proceedings. His research interests include all aspects of theoretical and applied computational electromagnetics. His principal research focus has been on the development of fast frequency- and time-domain integral-equation-based techniques for analyzing electromagnetic phenomena and the development of robust optimizers for the synthesis of electromagnetic/optical devices.

Prof. Michielssen is a member of the International Union of Radio Scientists (URSI) Commission B. He was the Technical Chairman of the 1997 Applied Computational Electromagnetics Society (ACES) Symposium (Review of Progress in Applied Computational Electromagnetics, March 1997, Monterey, CA). From 1998 to 2001 and from 2002 to 2003, he served on the ACES Board of Directors and, from 1998 to 2001, as the ACES Vice President. From 1998 to 2005, he served as an Associate Editor for the IEEE TRANSACTIONS ON Antennas and Propagation. He was also an Associate Editor for Radio Science. He was a recipient of the Belgian American Educational Foundation Fellowship in 1988, the Schlumberger Fellowship in 1990, the 1994 URSI Young Scientist Fellowship, the 1995 National Scientist Foundation CAREER Award, the 1998 Applied Computational Electromagnetics Society (ACES) Valued Service Award, the 1999 URSI Koga Gold Metal, and UIUC's 2001 Xerox Award for Faculty Research. In addition, he was named 1999 URSIUnited States National Committee Henry G. Booker Fellow, the 2002 Beckman Fellow of the UIUC Center for Advanced Studies, the 2003 Scholar of the Tel Aviv University Sackler Center for Advanced Studies, and selected as the UIUC 2003 University Scholar.

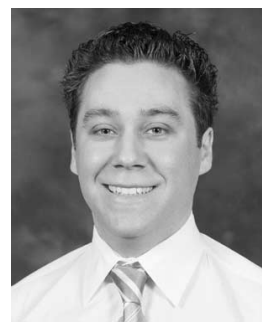

Anthony Grbic (M'06) received the B.A.Sc., M.A.Sc., and Ph.D. degrees in electrical engineering from the University of Toronto, Toronto, ON, Canada, in 1998, 2000, and 2005, respectively.

$\mathrm{He}$ is currently an Assistant Professor with the Radiation Laboratory, Department of Electrical Engineering and Computer Science, University of Michigan, Ann Arbor. His research interests include negative refractive index metamaterials at microwave and optical frequencies, periodic structures, printed antennas, and microwave circuits

Prof. Grbic was a recipient of the Best Student Paper Award at the 2000 Antenna Technology and Applied Electromagnetics Symposium and the IEEE Microwave Theory and Techniques Society Graduate Fellowship in 2001. 